\title{
THE CAUCHY PROBLEM FOR THE MAGNETO-HYDRODYNAMIC SYSTEM
}

\author{
MARCO CANNONE ${ }^{1}$, CHANGXING MIAO $^{2}$, NICOLAS PRIOUX ${ }^{3}$ \\ and BAOQUAN YUAN ${ }^{4}$ \\ ${ }^{1,3}$ Laboratoire d'Analyse et de Mathématiques Appliquées \\ Université de Marne-la-Vallée \\ Cité Descartes-5, bd Descartes, Champs-sur-Marne \\ 77454 Marne-la-Vallée, Cedex 2, France \\ E-mail: marco.cannone@univ-mlv.fr, nicolas.prioux@univ-mlv.fr \\ ${ }^{2}$ Institute of Applied Physics and Computational Mathematics \\ P.O. Box 8009, Beijing 100088, P.R. China \\ E-mail: miao_changxing@iapcm.ac.cn \\ ${ }^{4}$ Institute of Applied Mathematics, Academy of Mathematics \& Systems Science \\ Chinese Academy of Sciences, Beijing 100080, P.R. China \\ and \\ Henan Polytechnic University \\ Jiaozuo City, Henan Province, 454000, P.R. China \\ E-mail: bqyuan@hpu.edu.cn
}

\begin{abstract}
We study the uniqueness and regularity of Leray-Hopf's weak solutions for the MHD equations with dissipation and resistance in different frameworks. Using different kinds of spacetime estimates in conjunction with the Littlewood-Paley-Bony decomposition, we present some general criteria of uniqueness and regularity of weak solutions to the MHD system, and prove the uniqueness and regularity criterion in the framework of mixed space-time Besov spaces by applying Tao's trichotomy method.
\end{abstract}

2000 Mathematics Subject Classification: 76W05, 74H25, 74H30.

Key words and phrases: MHD system, space-time estimates, uniqueness and regularity, Leray-Hopf weak solutions, Bony's paraproduct decomposition, Tao's trichotomy decomposition, Besov spaces.

The research of Changxing Miao was partly supported by the National Natural Science Foundation (NNSF) of China No. 10571016. The research of Baoquan Yuan was partly supported by the NNSF of China and Natural Science Foundation of Henan Province No. 0611055500. The research of Marco Cannone and Nicolas Prioux has been supported by a Marie Curie Transfer of Knowledge Fellowship of the European Community's Sixth Framework Programme under contract number MTKD-CT-2004-013389.

The paper is in final form and no version of it will be published elsewhere. 
1. Introduction. In this paper we are concerned with the Cauchy problem for the MHD system with dissipation and resistance in $\mathbb{R}^{n}, n \geq 3$,

$$
\begin{aligned}
& \partial_{t} u+(u \cdot \nabla) u=-\nabla \Pi+(b \cdot \nabla) b+\nu \Delta u, \text { in } \quad \mathbb{R}^{n} \times(0, T), \\
& \partial_{t} b+(u \cdot \nabla) b=(b \cdot \nabla) u+\eta \Delta b, \text { in } \mathbb{R}^{n} \times(0, T), \\
& \nabla \cdot u=\nabla \cdot b=0, \text { in } \mathbb{R}^{n} \times(0, T), \\
& u(x, 0)=u_{0}(x), \quad b(x, 0)=b_{0}(x), \quad x \in \mathbb{R}^{n},
\end{aligned}
$$

where $u(x, t)$ is the flow velocity, $b(x, t)$ is the magnetic field, $\Pi=P+\frac{1}{2}|b|^{2}$ is the total pressure, $\nu>0$ is the kinematic viscosity and $\eta>0$ is the resistivity. For other forms of the MHD systems, such as the ideal MHD system and the ideal MHD system with the resistivity, one can refer to $[8,12,30,33]$.

For any prescribed $\left(u_{0}, b_{0}\right) \in L^{2}\left(\mathbb{R}^{n}\right)$ with $\nabla \cdot u_{0}(x)=\nabla \cdot b_{0}(x)=0$, the Cauchy problem (1.1)-(1.4) has been shown to possess at least one global $L^{2}$-weak solution $(u, b)$ with

$$
(u(x, t), b(x, t)) \in L^{\infty}\left([0, T) ; L^{2}\left(\mathbb{R}^{n}\right)\right) \cap L^{2}\left([0, T) ; H^{1}\left(\mathbb{R}^{n}\right)\right), \quad \forall T>0,
$$

see G. Duvaut and J. L. Lions [12] and M. Sermange and R. Temam [30]. By means of interpolation theorem, one easily sees that the above $L^{2}$-weak solution $(u, b)$ satisfies the following space-time integrability

$$
(u, b) \in L^{q}\left([0, T) ; L^{r}\left(\mathbb{R}^{n}\right)\right), \quad \forall \quad 0<T \leq \infty,
$$

where $(q, r) \in \Xi$, i.e.

$$
\frac{2}{q}=n\left(\frac{1}{2}-\frac{1}{r}\right), \quad 2 \leq r \leq 2^{*}=\frac{2 n}{n-2} .
$$

But $(u, b) \in L^{q}\left(I ; L^{r}\left(\mathbb{R}^{n}\right)\right),(q, r) \in \Xi$, cannot assure the uniqueness and regularity of the weak solutions for (1.1)-(1.4).

One natural problem is to find suitable conditions which assure the uniqueness and regularity of the weak solutions for the MHD equation (1.1)-(1.4). Similarly to the cases of Navier-Stokes equations [2, 4, 21], Wu in [33] proved a regularity result for general classical weak solutions for the MHD equations in $\mathbb{R}^{3}$ by energy integral estimates. More precisely, let $\left(u_{0}(x), b_{0}(x)\right) \in L^{p}\left(\mathbb{R}^{3}\right) \cap H^{1}\left(\mathbb{R}^{3}\right)$ with $p>3$, and assume that $(u(x, t), b(x, t)) \in$ $L^{\infty}\left([0, T) ; L^{2}\left(\mathbb{R}^{3}\right)\right) \cap L^{2}\left([0, T) ; H^{1}\left(\mathbb{R}^{3}\right)\right), T>0$, is a weak solution, then

$$
(u, b) \in C^{\infty}\left((0, T) \times \mathbb{R}^{3}\right)
$$

if $(u, b) \in L^{q}\left([0, T] ; L^{r}\left(\mathbb{R}^{3}\right)\right)$, where

$$
\frac{2}{q}+\frac{3}{r}=1, \quad r>3, \quad q \geq 2 .
$$

His regularity result is different from Serrin's regularity criterion because he added an artificial restriction on initial data. On the other hand, it seems that he used the restricted condition $r<7$ in [33], but it can be removed by the regularization method and extended to the case of $n$-dimensional spaces.

It is well-known that continuation of smooth solutions plays an important role in the study of the uniqueness and regularity of weak solutions in Navier-Stokes equations [17]-[19]. For this reason, many authors studied the continuation or blow-up criterion for 
the smooth solutions to the MHD system. For example, Caflisch, Klapper and Steel [5] extended the well-known result of Beale, Kato and Majda on the 3-dimensional Euler equation to the 3 -D ideal MHD equations $(\nu=\eta=0)$ and obtained the endpoint type continuation criterion for smooth solutions $(u, b)$, i.e.

$$
\int_{0}^{T}\|\omega\|_{\infty} d x<\infty, \quad \int_{0}^{T}\|j\|_{\infty} d t<\infty, \quad \omega=\nabla \times u, \quad j=\nabla \times b,
$$

which implies the smooth solution $(u, b)$ can be extended beyond $t=T$. Yuan [36, 37], Zhang and Liu in [38] studied the continuation or blow-up criterion of the smooth solutions to the MHD system and the ideal MHD system, respectively. They proved that smooth solutions $(u, b)$ can be extended beyond $t=T$ if

$$
\int_{0}^{T}\|\omega\|_{\dot{B}_{\infty, \infty}^{0}\left(\mathbb{R}^{3}\right)} d t<\infty, \quad \omega=\nabla \times u
$$

and

$$
\int_{0}^{T}\|j\|_{\dot{B}_{\infty, \infty}^{0}\left(\mathbb{R}^{3}\right)} d t<\infty, \quad j=\nabla \times b,
$$

for the ideal MHD system or the MHD system, respectively, where $\dot{B}_{\infty, \infty}^{0}\left(\mathbb{R}^{3}\right)$ denotes the homogeneous Besov space.

Many authors studied the uniqueness and regularity of the Leray-Hopf weak solutions to Navier-Stokes equations in different frameworks, see [2, 4, 9], [14]-[22], [24, 31]. Recently, Zhang and Chen in [39] applied Littlewood-Paley trichotomy to study the spacetime estimates for the Navier-Stokes equations and extended the well-known regularity criterion of weak solutions and blow-up criterion of the smooth solutions.

In this paper we will study the uniqueness and the regularity of Leray-Hopf's weak solutions for the MHD equations with dissipation and resistance under different frameworks.

Before stating the definition of Leray-Hopf's weak solutions for the MHD equations, we introduce some function spaces and notions. Let $C_{0, \sigma}^{\infty}$ denote the set of all $C^{\infty}$ vector functions $\left(\phi_{1}, \cdots, \phi_{n}\right)$ with compact support in $\mathbb{R}^{n}$, such that $\operatorname{div} \phi=0$. $L_{\sigma}^{p}$ is the closure of $C_{0, \sigma}^{\infty}$ with respect to the $L^{p}$-norm $\|\cdot\|_{p}$. For $1 \leq p \leq \infty, L^{p}$ stands for the usual (vector-valued) Lebesgue space over $\mathbb{R}^{n},(\cdot, \cdot)$ denotes the duality pairing between $L^{p}$ and $L^{p^{\prime}}$, where $\frac{1}{p}+\frac{1}{p^{\prime}}=1$. For $s \in \mathbb{R}, H^{s}\left(\mathbb{R}^{n}\right)$ stands for the usual (vector-valued) Hilbert spaces over $\mathbb{R}^{n}, H_{\sigma}^{s}$ denotes the closure of $C_{0, \sigma}^{\infty}$ with respect to the $H^{s}$-norm

$$
\|\phi\|_{H^{s}}=\left\|(1-\Delta)^{\frac{s}{2}} \phi\right\|_{2} .
$$

$\operatorname{BMO}\left(\mathbb{R}^{n}\right)$ stands for the bounded mean oscillation space, and $\mathcal{H}^{1}\left(\mathbb{R}^{n}\right)$ stands for the Hardy space, which is the dual space of $\operatorname{BMO}\left(\mathbb{R}^{n}\right)$. We take an arbitrary function $\varphi$ in the Schwartz class $\mathcal{S}\left(\mathbb{R}^{n}\right)$ and whose Fourier transform $\hat{\varphi}$ is such that $0 \leq \hat{\varphi} \leq 1$ and

$$
\begin{cases}\hat{\varphi}(\xi)=1, & \text { if }|\xi| \leq \frac{3}{4} \\ \hat{\varphi}(\xi)=0, & \text { if }|\xi| \geq \frac{4}{3}\end{cases}
$$

Let $\psi(x)=2^{n} \varphi(2 x)-\varphi(x)$ and

$$
\varphi_{j}(x)=2^{n j} \varphi\left(2^{j} x\right), \quad \psi_{j}=2^{j n} \psi\left(2^{j} x\right), \quad j \in \mathbb{Z} .
$$


We denote by $S_{j}$ and $\triangle_{j}$, respectively, the convolution operators with $\varphi_{j}$ and $\psi_{j}$, then the set $\left\{S_{j}, \triangle_{j}\right\}$ is the Littlewood-Paley decomposition of the unity

$$
I=S_{0}+\sum_{j \geq 0} \triangle_{j}, \quad S_{0} f=\varphi * f
$$

and the corresponding homogeneous decomposition of unity is

$$
I=\sum_{j \in \mathbb{Z}} \triangle_{j}, \quad \triangle_{j}=S_{j}-S_{j-1} .
$$

To define the homogeneous spaces we introduce the equivalence class $Z^{\prime}=S^{\prime} / P$, where $P$ is the set of polynomials, see [7, page 180] for details.

By means of the above Littlewood-Paley decomposition, Besov spaces can be defined as:

$$
\begin{gathered}
B_{p, q}^{s}=\left\{f \in S^{\prime}\left(\mathbb{R}^{n}\right), \quad\|f\|_{B_{p, q}^{s}}=\left\|S_{0} f\right\|_{p}+\left(\sum_{j=0}^{\infty} 2^{j s q}\left\|\Delta_{j} f\right\|_{p}^{q}\right)^{\frac{1}{q}}\right. \\
\left.=\|\varphi * f\|_{p}+\left(\sum_{j=1}^{\infty} 2^{j s q}\left\|\psi_{j} * f\right\|_{p}^{q}\right)^{\frac{1}{q}}<\infty\right\}, \\
\dot{B}_{p, q}^{s}=\left\{f \in Z^{\prime}\left(\mathbb{R}^{n}\right),\|f\|_{\dot{B}_{p, q}^{s}}=\left(\sum_{j \in \mathbb{Z}} 2^{j s q}\left\|\psi_{j} * f\right\|_{p}^{q}\right)^{\frac{1}{q}}<\infty\right\},
\end{gathered}
$$

where $1 \leq p \leq \infty, 1 \leq q \leq \infty, s \in \mathbb{R}$. Notice that for noninteger $s>0$, we have $B_{p, p}^{s}\left(\mathbb{R}^{n}\right)=W^{s, p}\left(\mathbb{R}^{n}\right)$, where $W^{s, p}\left(\mathbb{R}^{n}\right)=\left\{f \in \mathcal{S}^{\prime}\left(\mathbb{R}^{n}\right) ;\|f\|_{W^{s, p}}<\infty\right\}$ is the fractional ordered Sobolev space with norm

$$
\|f\|_{W^{s, p}}=\|f\|_{W^{[s], p}}+\sum_{|\alpha|=[s]}\left(\int_{\mathbb{R}^{n} \times \mathbb{R}^{n}} \frac{\left|D^{\alpha} f(x)-D^{\alpha} f(y)\right|^{p}}{|x-y|^{(s-[s]) p+n}} d x d y\right)^{\frac{1}{p}} .
$$

In the same way, we can define Triebel-Lizorkin spaces as follows:

$$
\begin{gathered}
F_{p, q}^{s}=\left\{f \in S^{\prime}\left(\mathbb{R}^{n}\right) ; \quad\|f\|_{F_{p, q}^{s}}=\|\varphi * f\|_{p}+\left\|\left(\sum_{j=1}^{\infty} 2^{j s q}\left|\psi_{j} * f\right|^{q}\right)^{\frac{1}{q}}\right\|_{p}<\infty\right\}, \\
\dot{F}_{p, q}^{s}=\left\{f \in Z^{\prime}\left(\mathbb{R}^{n}\right) ; \quad\|f\|_{\dot{F}_{p, q}^{s}}=\left\|\left(\sum_{j=1}^{\infty} 2^{j s q}\left|\psi_{j} * f\right|^{q}\right)^{\frac{1}{q}}\right\|_{p}<\infty\right\},
\end{gathered}
$$

where $s \in \mathbb{R}, 1 \leq p<\infty, 1 \leq q \leq \infty$. When $q=2$, we have $F_{p, 2}^{s}\left(\mathbb{R}^{n}\right)=H^{s, p}\left(\mathbb{R}^{n}\right)$, where $H^{s, p}\left(\mathbb{R}^{n}\right)=\left\{f \in \mathcal{S}^{\prime}\left(\mathbb{R}^{n}\right) ;\|f\|_{H^{s, p}}<\infty\right\}$ is the potential Banach space with the norm

$$
\|f\|_{H^{s, p}} \cong\left(\int_{\mathbb{R}^{n}}\left(1+|\xi|^{2}\right)^{s}|\hat{f}(\xi)|^{2} d \xi\right)^{\frac{1}{2}} .
$$

Moreover, $\dot{F}_{\infty, 2}^{0} \cong \operatorname{BMO}\left(\mathbb{R}^{n}\right)$ and $\mathcal{H}^{1} \cong \dot{F}_{1,2}^{0}\left(\mathbb{R}^{n}\right)$. Please refer to [3], [7], [9], [26] and [34] for more details about function spaces.

Definition 1.1. Let $\left(u_{0}(x), b_{0}(x)\right) \in L_{\sigma}^{2}\left(\mathbb{R}^{n}\right)$. A measurable function $(u, b)$ on $\mathbb{R}^{n} \times(0, T)$ is called a weak solution of (1.1)-(1.4) on $(0, T)$ if

(i) $(u, b) \in L^{\infty}\left((0, T) ; L_{\sigma}^{2}\right) \cap L^{2}\left((0, T) ; H_{\sigma}^{1}\right)$;

(ii) For every $(\Phi, \Psi) \in H^{1}\left((0, T) ; H_{\sigma}^{1} \cap L^{n}\right)$ with $\Phi(T)=\Psi(T)=0$, 


$$
\int_{0}^{T}\left\{-\left(u, \partial_{\tau} \Phi\right)+\nu(\nabla u, \nabla \Phi)+(u \cdot \nabla u, \Phi)-(b \cdot \nabla b, \Phi)\right\} d \tau=-\left(u_{0}, \Phi(0)\right),
$$

and

$$
\int_{0}^{T}\left\{-\left(b, \partial_{\tau} \Psi\right)+\eta(\nabla b, \nabla \Psi)+(u \cdot \nabla b, \Psi)-(b \cdot \nabla u, \Psi)\right\} d \tau=-\left(b_{0}, \Psi(0)\right) .
$$

By means of Galerkin approximation and compactness method, we have existence of the weak solutions, see G. Duvaut and J. L. Lions [12] and M. Sermange and R. Temam [30].

Proposition 1.1. For any prescribed $\left(u_{0}, b_{0}\right) \in L_{\sigma}^{2}\left(\mathbb{R}^{n}\right)$, the Cauchy problem (1.1)-(1.4) has been shown to possess at least one global $L^{2}$-weak solution $(u, b)$ on $(0, \infty)$ such that

$$
\begin{array}{r}
(u(x, t), b(x, t)) \in L^{\infty}\left([0, T) ; L_{\sigma}^{2}\left(\mathbb{R}^{n}\right)\right) \cap L^{2}\left([0, T) ; H^{1}\left(\mathbb{R}^{n}\right)\right), \quad \forall T>0, \\
\lim _{t \rightarrow 0}\left\|u(x, t)-u_{0}(x)\right\|_{2}=0, \quad \lim _{t \rightarrow 0}\left\|b(x, t)-b_{0}(x)\right\|_{2}=0,
\end{array}
$$

and energy inequality

$$
\begin{aligned}
& \int_{\mathbb{R}^{n}}\left(|u|^{2}+|b|^{2}\right) d x+2 \nu \int_{0}^{T} \int_{\mathbb{R}^{n}}|\nabla \otimes u|^{2} d x d t+2 \eta \int_{0}^{T} \int_{\mathbb{R}^{n}}|\nabla \otimes b|^{2} d x d t \\
\leq & \int_{\mathbb{R}^{n}}\left(\left|u_{0}\right|^{2}+\left|b_{0}\right|^{2}\right) d x,
\end{aligned}
$$

where

$$
\int_{\mathbb{R}^{n}}|u|^{2} d x=\int_{\mathbb{R}^{n}} \sum_{j=1}^{n}\left|u_{j}\right|^{2} d x, \quad|\nabla \otimes u|^{2}=\sum_{j=1}^{n} \sum_{k=1}^{n}\left|\partial_{k} u_{j}\right|^{2} .
$$

Usually, we call a weak solution $(u, b)$ in Definition 1.1 the Leray-Hopf weak solution of the MHD equation (1.1)-(1.4) if it satisfies energy inequality (1.17).

We are interested in the uniqueness and regularity of the Leray-Hopf weak solutions of the MHD equation (1.1)-(1.4) and related problems.

We conclude the introduction by giving some notations and some preliminary lemmas to be used throughout the paper. First, let us recall Bony's paraproduct of two tempered distributions $\pi(f, g)$ by means of the Littlewood-Paley decomposition. Formally, the product of two tempered distributions is decomposed into two Bony's paraproducts and a remainder term, i.e.

$$
\begin{aligned}
f g & =\pi(f, g)+\pi(g, f)+R(f, g) \\
& \triangleq \sum_{j \in \mathbb{Z}} \Delta_{j} f S_{j-2} g+\sum_{j \in \mathbb{Z}} \Delta_{j} g S_{j-2} f+\sum_{|j-k| \leq 1} \Delta_{j} f \Delta_{k} g .
\end{aligned}
$$

On the other hand, another form of Littlewood-Paley decomposition shares the same effect with Bony's decomposition. Tao calls this the Littlewood-Paley trichotomy

$$
\begin{aligned}
\Delta_{j}(f g)= & \Delta_{j}\left(S_{j-5} f \sum_{|l-j| \leq 3} \Delta_{l} g\right)+\Delta_{j}\left(\sum_{|l-j| \leq 3} \Delta_{l} f \cdot S_{j-5} g\right) \\
& +\Delta_{j}\left(\sum_{|l-j| \leq 5} \sum_{|k-j| \leq 5} \Delta_{l} f \Delta_{k} g\right)+\Delta_{j}\left(\sum_{l \text { or } k>j+5,|l-k| \leq 3} \Delta_{k} g \Delta_{l} f\right) .
\end{aligned}
$$


Let $B$ be a translation invariant Banach space which consists of distributions on $\mathbb{R}^{n}$. Usually, the space-time Banach space $L_{T}^{q}(B) \triangleq L^{q}(0, T ; B)$ for appropriate $B$ plays an important role in the study of PDEs. In particular, when $B=B_{p, \varrho}^{s}$ or $\dot{B}_{p, \varrho}^{s}, L^{q}\left(I ; B_{p, \varrho}^{s}\right)$ or $L^{q}\left(I ; \dot{B}_{p, \varrho}^{s}\right)$ represent usual space-time Banach spaces in the scale of Besov or homogeneous Besov spaces, where $(q, p, \varrho) \in[1, \infty]$ and $s \in \mathbb{R}$. For the sake of application, we introduce the following homogeneous mixed space-time Besov spaces

$$
\begin{aligned}
\mathcal{L}^{q}\left(I ; \dot{B}_{p, \varrho}^{s}\left(\mathbb{R}^{n}\right)\right)=\{u(x, t) & \in \mathcal{D}^{\prime}\left(I ; \mathcal{S}^{\prime}\left(\mathbb{R}^{n}\right)\right) \\
& \left.\|u\|_{\mathcal{L}^{q}\left(I ; \dot{B}_{p, \varrho}^{s}\right)}=\left(\sum_{j \in \mathbb{Z}} 2^{j s \varrho}\left\|\triangle_{j} u\right\|_{L^{q}\left(I ; L^{p}\left(\mathbb{R}^{n}\right)\right)}^{\varrho}\right)^{\frac{1}{\varrho}}<\infty\right\} .
\end{aligned}
$$

which were considered in [11].

Lemma $1.1([3,7,9,23])$. Let $1 \leq p \leq \infty, f(x) \in \mathcal{S}^{\prime}\left(\mathbb{R}^{n}\right)$ with $\operatorname{supp} \hat{f} \sim 2^{j}$. Then

$$
\begin{array}{r}
\|f(x)\|_{p} \leq C 2^{-j|\alpha|}\left\|\partial^{\alpha} f\right\|_{p}, \quad 1 \leq p \leq \infty, \\
\left\|\partial^{\alpha} f\right\|_{q} \leq C 2^{j n\left(\frac{1}{p}-\frac{1}{q}\right)+j|\alpha|}\|f\|_{p}, \quad 1 \leq p \leq q \leq \infty .
\end{array}
$$

Lemma $1.2([21,28])$. Let $2 \leq p<\infty$. There exist two constants $C_{p} \geq c_{p}>0$ so that for every $f(x) \in \mathcal{S}^{\prime}\left(\mathbb{R}^{n}\right)$ with $\operatorname{supp} \hat{f} \sim 2^{j}, j \in \mathbb{Z}$. Then

$$
c_{p} 2^{2 j}\|f\|_{p}^{p} \leq\left\|\nabla\left(|f|^{\frac{p}{2}}\right)\right\|_{2}^{2} \sim \int_{\mathbb{R}^{n}}|\nabla f|^{2}|f|^{p-2} d x \leq C_{p} 2^{2 j}\|f\|_{p}^{p} .
$$

LEMMA $1.3([23,29])$. Let $k \in \mathbb{Z}, p, q$ and $r$ satisfy

$$
\frac{1}{p}=\frac{1}{q}+\frac{1}{r}, \quad 1 \leq p, q, r \leq \infty .
$$

Then there exists a constant $C>0$ such that

$$
\left\|\triangle_{k}(f \nabla g)-f \triangle_{k}(\nabla g)\right\|_{p} \leq C\|\nabla f\|_{q}\|g\|_{r} .
$$

Lemma $1.4([19,38])$. Let $1<p<\infty$. For $f, g \in W^{s, p}\left(\mathbb{R}^{n}\right)$, and $1<q \leq \infty, 1<r<\infty$, we have

$$
\left\|\nabla^{\alpha}(f g)-f \nabla^{\alpha} g\right\|_{p} \leq C\|\nabla f\|_{q}\left\|\nabla^{\alpha-1} g\right\|_{r}+\|g\|_{q}\left\|\nabla^{\alpha} f\right\|_{r},
$$

where $1 \leq \alpha \leq s$ and $\frac{1}{p}=\frac{1}{q}+\frac{1}{r}$.

In this paper, we shall study the regularity criterion (or the blow-up criterion) of the Leray-Hopf weak solutions in different frameworks, based on the space-time regularity of the heat equation and Bony's paraproduct decomposition. For this purpose, we first introduce some notations.

Definition 1.2. (i) Let $0 \leq \gamma \leq 1$, we say that $(q, r) \in \Lambda_{\gamma}$ if

$$
\frac{2}{q}=n\left(\frac{1+\gamma}{n}-\frac{1}{r}\right), \quad \frac{n}{1+\gamma} \leq r \leq \frac{n^{2}}{(1+\gamma) n-2} .
$$

(ii) Let $0 \leq \gamma \leq 1$, we say $(q, r) \in \tilde{\Lambda}_{\gamma}$ if

$$
\frac{2}{q}=n\left(\frac{1+\gamma}{n}-\frac{1}{r}\right), \quad \frac{n}{1+\gamma} \leq r \leq \infty .
$$


Usually we denote $\Lambda \triangleq \Lambda_{0}$ and $\tilde{\Lambda} \triangleq \tilde{\Lambda}_{0}$ when $\gamma=0$. We shall prove that the Leray-Hopf weak solution $(u, b)$ associated with initial data $\left(u_{0}, b_{0}\right)$ is unique if there exists $(q, r) \in \tilde{\Lambda}$ such that $(u, b) \in L^{q}\left(I ; L^{r}\left(\mathbb{R}^{n}\right)\right)$, see Section 4 for details.

2. Time-space estimates, mild and smooth solutions. Given $\left(u_{0}, b_{0}\right) \in X \subset$ $\mathcal{S}^{\prime}\left(\mathbb{R}^{n}\right)$ with $n \geq 2$, in order to find the solutions of (1.1)-(1.4), a natural approach is to solve the corresponding integral form by iterating the transform

$$
\left\{\begin{array}{l}
u(t) \mapsto e^{\nu \Delta t} u_{0}(x)+\int_{0}^{t} e^{\Delta(t-\tau)} \mathcal{P} \operatorname{div}(-u \otimes u+b \otimes b) d \tau, \\
b(t) \mapsto e^{\eta \Delta t} b_{0}(x)+\int_{0}^{t} e^{\eta \Delta(t-\tau)} \mathcal{P} \operatorname{div}(-u \otimes b+b \otimes u) d \tau,
\end{array}\right.
$$

where $f \otimes g$ is the tensor with $j k$-components $f_{j} g_{k}$ and $\partial \cdot(f \otimes g)$ is the vector with $j$-component $\partial_{k}\left(f_{j} g_{k}\right), \mathcal{P}$ denotes

$$
\mathcal{P} v=v-\nabla \frac{1}{\Delta}(\nabla \cdot v) .
$$

Usually, we define mild solutions the solutions obtained through the iterative transform (2.1) with the initial step $\left(u^{(0)}(t), b^{(0)}(t)\right)=\left(e^{\nu \Delta t} u_{0}, e^{\eta \Delta t} b_{0}\right)$. We call a space $\mathcal{X}(I)=C(I ; X) \cap Y$, on which we may apply the Picard contraction principle, an admissible resolution space for the MHD equations, $Y$ is some suitable space-time Banach spaces or weighted space-time Banach space. When $X=L_{\sigma}^{p}\left(\mathbb{R}^{n}\right), p \geq n$ (in the same way one can deal with the case $\left.X=H_{\sigma}^{s}\left(\mathbb{R}^{n}\right), s \geq \frac{n}{2}-1\right)$, it is easy to verify that the mild solutions become smooth solutions for $t>0$.

In this section we establish the well-posedness of mild solutions for (1.1)-(1.4) when we choose initial function spaces as $X=L_{\sigma}^{p}\left(\mathbb{R}^{n}\right), p \geq n$ or $X=H_{\sigma}^{s}\left(\mathbb{R}^{n}\right), s \geq \frac{n}{2}-1$. These results are similar to the results of Fujita-Kato [14] and Kato [16] or Giga [15] for Navier-Stokes equations. For the sake of completeness, we give a simple proof by space-time estimates approach, see $[25,26]$ for details. To this end, we introduce some notations.

Definition 2.1. We say $(q, r, p)$ is an admissible triplet if

$$
\frac{2}{q}=n\left(\frac{1}{p}-\frac{1}{r}\right), \quad q \triangleq q(r, p),
$$

where

$$
1<p \leq r< \begin{cases}\frac{n p}{n-2}, & n>2, \\ \infty, & n \leq 2 .\end{cases}
$$

We call $(q, r, p)$ a generalized admissible triplet if it satisfies (2.3) and

$$
1<p \leq r< \begin{cases}\frac{n p}{n-2 p}, & n>2 p \\ \infty, & n \leq 2 p\end{cases}
$$

REMARK 2.1. If $(q, r, p)$ is an admissible triplet, then $p<q \leq \infty$; if $(q, r, p)$ is a generalized admissible triplet, then $1<q \leq \infty$.

Now we introduce the following weighted space-time spaces which were defined in [16], see also [15], [25]-[27]. 
Definition 2.2. Let $B$ be a Banach space, $\varrho>0, I=[0, T)$ or $I=[0, \infty)$. Let $\dot{I}$ be an open interval with respect to $I$. Define $\mathcal{C}_{\varrho}(I ; B)$ and its corresponding homogeneous space $\dot{\mathcal{C}}_{\varrho}(I ; B)$ as

$$
\begin{aligned}
& \mathcal{C}_{\varrho}(I ; B)=\left\{f(t, x) \in C(\dot{I} ; B) ;\|f\|_{\mathcal{C}_{\varrho}(I ; B)} \triangleq \sup _{t \in I} t^{\frac{1}{\varrho}}\|f\|_{B}<\infty\right\}, \\
& \dot{\mathcal{C}}_{\varrho}(I ; B)=\left\{f(t, x) ; f(t, x) \in \mathcal{C}_{\varrho}(I ; B) \text { and } \lim _{t \rightarrow 0^{+}} t^{\frac{1}{\varrho}}\|f\|_{B}=0\right\} .
\end{aligned}
$$

REMARK 2.2. (i) Let $B=L^{p}, 1<p<\infty$, one easily verifies that $f(t, x) \in \mathcal{C}_{\varrho}\left(I ; L^{p}\right)$ if and only if $t^{\frac{1}{\varrho}} f \in C_{b}\left(I ; L^{p}\right)$.

(ii) Let $(q, p, r)$ be a generalized admissible triplet, then

$$
\mathcal{C}_{q(p, r)}\left(I ; L^{p}\right)=\left\{f(t, x) \in C\left(\dot{I} ; L^{p}\right) ;\|f\|_{\mathcal{C}_{q(p, r)}\left(I ; L^{p}\right)}=\sup _{t \in I} t^{\frac{1}{q}}\|f\|_{p}<\infty\right\},
$$

in particular,

$$
\mathcal{C}_{q(p, r)}\left(I ; L^{p}\right)=C_{b}\left(I ; L^{r}\right), \quad p=r .
$$

Let $S(t)=e^{A t}, A=\mathcal{P} \Delta, \mathcal{P}$ the projection operator from $\left(L^{p}\left(\mathbb{R}^{n}\right)\right)^{n}$ into $L_{\sigma}^{p}\left(\mathbb{R}^{n}\right)=$ $\left\{\varphi(x) \in\left(L^{p}\left(\mathbb{R}^{n}\right)\right)^{n}, \nabla \cdot \varphi(x)=0\right\}$, divergence-free vectors along gradients. By making use of multiplier estimates in conjunction with the theorem on singular integral operators $[13,21,26,32]$, we get the following $L^{p}-L^{r}$ estimates for $r \geq p>1$,

$$
\begin{gathered}
\left\|e^{-A t} \varphi\right\|_{r} \leq C t^{-\frac{n}{2}\left(\frac{1}{p}-\frac{1}{r}\right)}\|\varphi\|_{p}, \quad \forall t>0, \\
\left\|\nabla \mathcal{P} e^{-A t} \varphi\right\|_{r} \leq C t^{-\frac{1}{2}-\frac{n}{2}\left(\frac{1}{p}-\frac{1}{r}\right)}\|\varphi\|_{p}, \quad \forall t>0 .
\end{gathered}
$$

As an immediate consequence of the Young inequality and Marcinkiewicz interpolation theorem, we have

Proposition 2.1. (i) Let $(q, r, p)$ be any admissible triplet, $\varphi(x) \in L_{\sigma}^{p}$. Then $v(x, t)=$ $e^{-t A} \varphi \in L^{q}\left(I ; L^{r}\right) \cap C_{b}\left(I ; L_{\sigma}^{p}\right)$ with $\nabla \cdot v(x, t)=0$ and

$$
\left\|e^{-t A} \varphi\right\|_{L^{q}\left(I ; L^{r}\left(\mathbb{R}^{n}\right)\right)} \leq C\|\varphi\|_{p}, \quad I=[0, T), \quad 0<T \leq \infty .
$$

(ii) Let $(q, r, p)$ be any generalized admissible triplet, $\varphi(x) \in L_{\sigma}^{p}$. Then $v(t)=e^{-A t} \varphi \in$ $\mathcal{C}_{q(r, p)}\left(I ; L^{r}\left(\mathbb{R}^{n}\right)\right)$ such that $\nabla \cdot v=0$ and

$$
\left\|e^{-A t} \varphi ; \mathcal{C}_{q(r, p)}\left(I ; L^{r}\right)\right\| \leq C\|\varphi\|_{p}
$$

Moreover, if $(q, r, p)$ is a generalized admissible triplet with $p<r<\infty$, then $S(t) \varphi \in$ $\dot{\mathcal{C}}_{q(p, r)}\left(I, L^{r}\right)$, i.e.

$$
\lim _{t \rightarrow 0} t^{\frac{1}{q}}\|S(t) \varphi\|_{r}=0
$$

where $I=[0, T)$ or $[0, \infty)$.

See $[15]$ or $[25]-[27]$ for the proof.

Proposition 2.2. Let $p \geq p_{c}=n, I=[0, T),(q, r, p)$ be any generalized admissible triplet. Then

$$
G f \triangleq \int_{0}^{t} S(t-\tau) \mathcal{P} \nabla f d \tau
$$


satisfies the following estimates

$$
\begin{array}{r}
\|G f\|_{L^{q}\left(I ; L^{r}\right)}+\|G f\|_{L^{\infty}\left(I ; L^{p}\right)} \leq C T^{\frac{1}{2}-\frac{n}{2 p}}\|f\|_{L^{\frac{q}{2}}\left(I ; L^{\frac{r}{2}}\right)}, \quad r \leq 2 p, \\
\|G f\|_{\mathcal{C}_{q(r, p)}\left(I ; L^{r}\right)}+\|G f\|_{L^{\infty}\left(I ; L^{p}\right)} \leq C T^{\frac{1}{2}-\frac{n}{2 p}}\|f\|_{\mathcal{C}_{\frac{q}{2}\left(I ; L^{\frac{r}{2}}\right)},} r \leq 2 p, \\
\|G f\|_{L^{q}\left(I ; L^{r}\right)}+\|G f\|_{L^{\infty}\left(I ; L^{p}\right)} \leq C T^{\frac{1}{2}-\frac{n}{2 p}}\|f\|_{L^{\frac{q}{2}\left(I ; L^{\frac{r}{2}}\right)}}^{1-\theta}\|f\|_{L^{\infty}\left(I ; L^{\frac{p}{2}}\right)}^{\theta}, \quad r>2 p,
\end{array}
$$

and

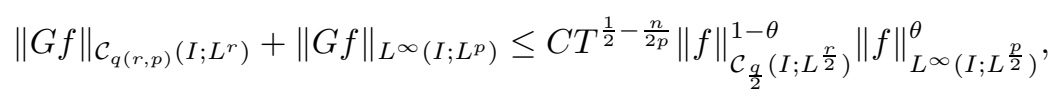

where $r>2 p$ and $\theta=\frac{r-2 p}{2(r-p)} \in(0,1)$.

Proof. We first prove (2.14). Since $r \leq 2 p$, one has, by the Hölder inequality or Young inequality,

$$
\begin{aligned}
& \|G f\|_{L^{\infty}\left(I ; L^{p}\right)} \leq C \int_{0}^{t}(t-s)^{-\frac{1}{2}-\frac{n}{2}\left(\frac{2}{r}-\frac{1}{p}\right)}\|f(s, x)\|_{\frac{r}{2}} d s \\
& \leq C\left(\int_{0}^{t}(t-s)^{-\left\{\frac{1}{2}+\frac{n}{2}\left(\frac{2}{r}-\frac{1}{p}\right)\right\} \chi_{1}} d s\right)^{\frac{1}{\chi_{1}}}\|f\|_{L^{\frac{q}{2}}\left(I ; L^{\frac{r}{2}}\right)} \\
& \leq C T^{\frac{1}{2}-\frac{n}{2 p}}\|f\|_{L^{\frac{q}{2}}\left(I ; L^{\frac{r}{2}}\right)}, \\
& \|G f\|_{L^{q}\left(I ; L^{r}\right)} \leq C\left\|\int_{0}^{t}(t-s)^{-\frac{1}{2}-\frac{n}{2}\left(\frac{2}{r}-\frac{1}{r}\right)}\right\| f(s, x)\left\|_{\frac{r}{2}} d s\right\|_{L^{q}} \\
& \leq C\left(\int_{0}^{T} t^{-\left\{\frac{1}{2}+\frac{n}{2 r}\right\} \chi_{2}} d t\right)^{\frac{1}{\chi_{2}}}\|f\|_{L^{\frac{q}{2}\left(I ; L^{\frac{r}{2}}\right)}} \\
& \leq C T^{\frac{1}{2}-\frac{n}{2 p}}\|f(s, x)\|_{L^{\frac{q}{2}}\left(I ; L^{\frac{r}{2}}\right)},
\end{aligned}
$$

where $\frac{1}{\chi_{1}}=1-\frac{2}{q}, 1+\frac{1}{q}=\frac{2}{q}+\frac{1}{\chi_{2}}$, and $C=C(n, p, r)$.

On the other hand, for the case $r>2 p$, by means of the Riesz interpolation theorem, Hölder inequality and Young inequality, we have

$$
\begin{aligned}
\|G f\|_{L^{\infty}\left(I ; L^{p}\right)} & \leq \int_{0}^{t}(t-s)^{-\frac{1}{2}}\left\||f(s, x)|^{\frac{1}{2}}\right\|_{2 p}^{2} d s \\
& =C \int_{0}^{t}(t-s)^{-\frac{1}{2}}\left\||f(s, x)|^{\frac{1}{2}}\right\|_{L^{p}}^{2 \theta}\left\||f(s, x)|^{\frac{1}{2}}\right\|_{r}^{2(1-\theta)} d s \\
& =C\left(\int_{0}^{t}(t-s)^{-\frac{1}{2} \chi_{1}} d s\right)^{\frac{1}{\chi 1}}\left\||f(s, x)|^{\frac{1}{2}}\right\|_{C\left(I ; L^{p}\right)}^{2 \theta}\left\||f(s, x)|^{\frac{1}{2}}\right\|_{L^{q}\left(I ; L^{r}\right)}^{2(1-\theta)} \\
& \leq C T^{\frac{1}{2}-\frac{2(1-\theta)}{q}}\left\||f(s, x)|^{\frac{1}{2}}\right\|_{C\left(I ; L^{p}\right)}^{2 \theta}\left\||f(s, x)|^{\frac{1}{2}}\right\|_{L^{q}\left(I ; L^{r}\right)}^{2(1-\theta)} \\
& \leq C T^{\frac{1}{2}-\frac{n}{2 p}}\|f(s, x)\|_{C\left(I ; L^{\frac{p}{2}}\right)}^{\theta}\|f(s, x)\|_{L^{\frac{q}{2}}\left(I ; L^{\frac{r}{2}}\right)}^{(1-\theta)} \\
\|G f\|_{L^{q}\left(I ; L^{r}\right)} & \leq C\left\|\int_{0}^{t}(t-s)^{-\frac{1}{2}-\frac{n}{2}\left(\frac{1}{p}-\frac{1}{r}\right)}\right\||f(s, x)|^{\frac{1}{2}}\left\|_{2 p}^{2} d s\right\|_{q} \\
& \leq C\left\|\int_{0}^{t}(t-s)^{-\frac{1}{2}-\frac{n}{2}\left(\frac{1}{p}-\frac{1}{r}\right)}\right\||f(s, x)|^{\frac{1}{2}}\left\|_{p}^{2 \theta}\right\||f(s, x)|^{\frac{1}{2}}\left\|_{r}^{2(1-\theta)} d s\right\|_{q}
\end{aligned}
$$




$$
\begin{aligned}
& \leq C\left(\int_{0}^{T} t^{-\left\{\frac{1}{2}+\frac{n}{2}\left(\frac{1}{p}-\frac{1}{r}\right)\right\} \chi_{2}} d t\right)^{\frac{1}{\chi_{2}}}\left\||f(s, x)|^{\frac{1}{2}}\right\|_{C\left(I ; L^{p}\right)}^{2 \theta}\left\||f(s, x)|^{\frac{1}{2}}\right\|_{L^{q}\left(I ; L^{r}\right)}^{2(1-\theta)} \\
& \leq C T^{\frac{1}{2}-\frac{n}{2 p}}\left\||f(s, x)|^{\frac{1}{2}}\right\|_{C\left(I ; L^{p}\right)}^{2 \theta}\left\||f(s, x)|^{\frac{1}{2}}\right\|_{L^{q}\left(I ; L^{r}\right)}^{2(1-\theta)} \\
& \leq C T^{\frac{1}{2}-\frac{n}{2 p}}\|f(s, x)\|_{C\left(I ; L^{\frac{p}{2}}\right)}^{\theta}\|f(s, x)\|_{L^{\frac{q}{2}}\left(I ; L^{\frac{r}{2}}\right)}^{(1-\theta)},
\end{aligned}
$$

where $\theta$ satisfies $\frac{1}{2 p}=\frac{\theta}{p}+\frac{1-\theta}{r}$ and

$$
1=\frac{2(1-\theta)}{q}+\frac{1}{\chi_{1}}, \quad 1+\frac{1}{q}=\frac{2(1-\theta)}{q}+\frac{1}{\chi_{2}} .
$$

One easily checks that

$$
\frac{1}{2}-\frac{2(1-\theta)}{q}=\frac{1}{2}-\frac{r}{q(r-p)}=\frac{1}{2}-\frac{n}{2 p}
$$

and

$$
\frac{1}{\chi_{2}}-\frac{1}{2}-\frac{n}{2}\left(\frac{1}{p}-\frac{1}{r}\right)=\frac{1}{2}-\frac{2(1-\theta)}{q}=\frac{1}{2}-\frac{n}{2 p},
$$

Arguing similarly to (2.14) and (2.16), we can prove the estimates (2.15) and (2.17).

By applying the orthogonal projector operator

$$
\mathcal{P}: L^{p}\left(\mathbb{R}^{n}\right) \rightarrow L_{\sigma}^{p}\left(\mathbb{R}^{n}\right)
$$

to both sides of (1.1)-(1.4) with $\nabla \cdot u_{0}(x)=\nabla \cdot b_{0}(x)=0$, we have

$$
\left\{\begin{array}{l}
\partial_{t} u+\nu A u=-\mathcal{P} \partial \cdot(u \otimes u)+\mathcal{P} \partial \cdot(b \otimes b) \\
\partial_{t} b+\eta A b=-\mathcal{P} \partial \cdot(u \otimes b)+\mathcal{P} \partial \cdot(b \otimes u) \\
u(0)=u_{0}(x), \quad b(0)=b_{0}(x)
\end{array}\right.
$$

which is equivalent to the following system of integral equations

$$
\left\{\begin{array}{l}
u(t)=e^{-\nu A t} u_{0}(x)+\int_{0}^{t} e^{-A(t-\tau)} \mathcal{P} \operatorname{div}(-u \otimes u+b \otimes b) d \tau, \\
b(t)=e^{-\eta A t} b_{0}(x)+\int_{0}^{t} e^{-\eta A(t-\tau)} \mathcal{P} \operatorname{div}(-u \otimes b+b \otimes u) d \tau,
\end{array}\right.
$$

where $A$ generates an analytic operator semigroup in $L^{p}$ with divergence-free vectors, where $1<p<\infty$.

Applying Proposition 2.1, Proposition 2.2, the regularity of parabolic equations and standard contraction principle to $(2.19)$ in resolution spaces $L^{q}\left(I ; L^{r}\left(\mathbb{R}^{n}\right)\right) \cap C\left(I ; L_{\sigma}^{p}\left(\mathbb{R}^{n}\right)\right)$ or $\mathcal{C}_{q(p, r)}\left(I ; L^{r}\left(\mathbb{R}^{n}\right)\right) \cap C\left(I ; L_{\sigma}^{p}\left(\mathbb{R}^{n}\right)\right)$, we have

TheOREM 2.1. (i) Let $p \geq p_{c}=n, u_{0}(x) \in L_{\sigma}^{p}$ and $b_{0}(x) \in L_{\sigma}^{p}$. Assume that $(q, r, p)$ is any admissible triplet, then there exist $T^{*}>0$ and a unique solution to (2.19) such that

$$
\begin{aligned}
& (u(t), b(t)) \in C\left(\left[0, T^{*}\right) ; L_{\sigma}^{p}\right) \cap L^{q}\left(\left(0, T^{*}\right) ; L^{r}\left(\mathbb{R}^{n}\right)\right), \\
& \text { for } T^{*}=T\left(\left\|u_{0}\right\|_{p}+\left\|b_{0}\right\|_{p}\right), p>n,
\end{aligned}
$$

or

$$
\left.(u(t), b(t)) \in C\left(\left[0, T^{*}\right) ; L_{\sigma}^{p}\right) \cap L^{q}\left(\left(0, T^{*}\right) ; L^{r}\left(\mathbb{R}^{n}\right)\right)\right), T^{*}=T\left(u_{0}, b_{0}\right), p=n .
$$


(ii) Let $p>n$. If $T^{*}<\infty$, then

$$
\lim _{t \rightarrow T^{*}}\left(\|u(t)\|_{r}+\|b(t)\|_{r}\right)=\infty, \quad p \leq r \leq \infty
$$

moreover,

$$
\|u(t)\|_{r}+\|b(t)\|_{r} \geq \frac{C}{\left(T^{*}-t\right)^{\frac{1}{2}-\frac{n}{2 r}}} .
$$

(iii) Let $p=n$, then $T^{*}=\infty$ provided that $\left\|u_{0}\right\|_{n}+\|b\|_{n} \ll 1$.

(iv) $(u(t), b(t)) \in C^{\infty}\left(\left(0, T^{*}\right) \times \mathbb{R}^{n}\right)$.

Theorem 2.2. Let $p \geq p_{c}=n, u_{0}(x) \in L_{\sigma}^{p}$ and $b_{0}(x) \in L_{\sigma}^{p}$. Assume that $(q, r, p)$ is any generalized admissible triplet, then there exist $T^{*}>0$ and a unique solution to (2.19) such that

$$
(u(t), b(t)) \in C\left(\left[0, T^{*}\right) ; L_{\sigma}^{p}\right) \cap \mathcal{C}_{q(r, p)}\left(\left(0, T^{*}\right) ; L^{r}\right), T^{*}=T\left(\left\|u_{0}\right\|_{p},\left\|b_{0}\right\|_{p}\right),
$$

for $p>n$, or

$$
(u(t), b(t)) \in C\left(\left[0, T^{*}\right) ; L_{\sigma}^{p}\right) \cap \mathcal{C}_{q(r, p)}\left(\left(0, T^{*}\right) ; L^{r}\right), \quad T^{*}=T\left(u_{0}, b_{0}\right), \quad p=n,
$$

and (ii), (iii), (iv) in Theorem 2.1 also hold.

3. The mild solutions in Besov spaces. In this section we establish the well-posedness of the Cauchy problem (1.1)-(1.4) for initial data in Besov spaces by means of the space-time mixed Besov spaces. First we give preliminary linear and nonlinear estimates.

Proposition 3.1. Let $q, \varrho \in[1, \infty], 2 \leq p<\infty, s \in \mathbb{R}$. Assume that $v(x, t)$ is a solution of the Cauchy problem for the heat equation

$$
\left\{\begin{array}{l}
\partial_{t} v+\Delta v=f(x, t), \quad(x, t) \in \mathbb{R}^{n} \times \mathbb{R}^{+}, \\
v(x, 0)=v_{0}(x), \quad x \in \mathbb{R}^{n} .
\end{array}\right.
$$

Then there exists a constant $C>0$ depending only on $n, q, p, \varrho$ such that, for all $0<T \leq$ $+\infty$,

$$
\|v\|_{\mathcal{L}^{q}\left(I ; \dot{B}_{p, \varrho}^{s+\frac{2}{q}}\left(\mathbb{R}^{n}\right)\right)} \leq C\left(\left\|v_{0}\right\|_{\dot{B}_{p, \varrho}^{s}}+\|f\|_{\mathcal{L}^{\ell}\left(I ; \dot{B}_{p, \varrho}^{s+\frac{2}{\ell}-2}\right)}\right), \quad 1 \leq \ell \leq q
$$

where $I=[0, T]$. In particular, when $q \geq \varrho$, we have by Minkowski inequality

$$
\|v\|_{L^{q}\left(I ; \dot{B}_{p, \varrho}^{s+\frac{2}{q}}\left(\mathbb{R}^{n}\right)\right)} \leq C\left(\left\|v_{0}\right\|_{\dot{B}_{p, \varrho}^{s}}+\|f\|_{\mathcal{L}^{\ell}\left(I ; \dot{B}_{p, \varrho}^{s+\frac{2}{\ell}-2}\right)}\right), \quad 1 \leq \ell \leq q .
$$

Proof. Applying $\triangle_{j}$ to both sides of (3.1) and then multiplying both sides of this equation by $\left|\triangle_{j} v\right|^{p-2} \triangle_{j} v$, we get

$$
\frac{d}{d t} \triangle_{j} v \cdot\left|\triangle_{j} v\right|^{p-2} \triangle_{j} v-\Delta \triangle_{j} v \cdot\left|\triangle_{j} v\right|^{p-2} \Delta_{j} v=\triangle_{j} f \cdot\left|\Delta_{j} v\right|^{p-2} \triangle_{j} v .
$$

We integrate the both sides of (3.4) and apply the divergence theorem to obtain

$$
\frac{1}{p} \frac{d}{d t}\left\|\triangle_{j} v\right\|_{p}^{p}+\int_{\mathbb{R}^{n}} \nabla \triangle_{j} v \cdot \nabla\left(\left|\triangle_{j} v\right|^{p-2} \triangle_{j} v\right) d x \leq\left\|\triangle_{j} f\right\|_{p}\left\|\triangle_{j} v\right\|_{p}^{p-1} .
$$


Since

$$
\begin{aligned}
\int_{\mathbb{R}^{n}} \nabla \triangle_{j} v \cdot \nabla\left(\left|\triangle_{j} v\right|^{p-2} \triangle_{j} v\right) d x & =\frac{4(p-1)}{p^{2}} \int_{\mathbb{R}^{n}}\left|\triangle_{j} v\right|^{p-2}\left|\nabla \triangle_{j} v\right|^{2} d x \\
& =\int_{\mathbb{R}^{n}}\left|\nabla\left(\left|\triangle_{j} v\right|^{\frac{p}{2}}\right)\right|^{2} d x \geq c_{p} 2^{2 j}\left\|\triangle_{j} v\right\|_{p}^{p},
\end{aligned}
$$

we have

$$
\frac{d}{d t}\left\|\triangle_{j} v\right\|_{p}+2^{2 j} c_{p}\left\|\triangle_{j} v\right\|_{p} \leq\left\|\triangle_{j} f\right\|_{p}
$$

We integrate both sides of (3.7) with respect to $t$ to get

$$
\left\|\triangle_{j} v\right\|_{p} \leq e^{-2^{2 j} c_{p} t}\left\|\triangle_{j} v_{0}(x)\right\|_{p}+e^{-2^{2 j} c_{p} t} *\left\|\triangle_{j} f\right\|_{p}
$$

We take the $L^{q}$-norm with respect to $t$ on interval $I$ of both sides of (3.8) and by the Young inequality one has

$$
\left\|\triangle_{j} v\right\|_{L^{q}\left(I ; L^{p}\left(\mathbb{R}^{n}\right)\right)} \leq 2^{-\frac{2 j}{q}} c_{p}^{-\frac{1}{q}}\left\|\triangle_{j} v_{0}(x)\right\|_{p}+2^{-2 j\left(\frac{1}{q}-\frac{1}{\ell}+1\right)} c_{p}^{-\left(\frac{1}{q}-\frac{1}{\ell}+1\right)}\left\|\triangle_{j} f\right\|_{L^{\ell}\left(I ; L^{p}\right)},
$$

which immediately implies

$$
2^{j s+\frac{2 j}{q}}\left\|\triangle_{j} v\right\|_{L^{q}\left(I ; L^{p}\right)} \leq C\left[2^{j s}\left\|\triangle_{j} v_{0}(x)\right\|_{p}+2^{j\left(s+\frac{2}{\ell}-2\right)}\left\|\triangle_{j} f\right\|_{L^{\ell}\left(I ; L^{p}\right)}\right] .
$$

Now we take the $\ell^{\varrho}$-norm of both sides of (3.10) and arrive at (3.2). We thus complete the proof of Proposition 3.1.

Using Bony's paraproduct decomposition we estimate now the nonlinear term. Consider two tempered distributions $u(x, t)$ and $v(x, t)$, then

$$
u v=T_{u} v+T_{v} u+R(u, v) .
$$

First we deal with the paraproduct terms $T_{u} v$ or $T_{v} u$, in virtue of the Hölder inequality and noting the equivalent definition of negative index Besov space

$$
\left(\sum_{j \in \mathbb{Z}} 2^{j s r}\left\|S_{j} u\right\|_{L^{q}\left(I ; L^{p}\right)}^{r}\right)^{1 / r} \simeq\left(\sum_{j \in \mathbb{Z}} 2^{j s r}\left\|\triangle_{j} u\right\|_{L^{q}\left(I ; L^{p}\right)}^{r}\right)^{1 / r},
$$

for $s<0$ (cf. [7]), we have the following lemma.

LEMma 3.1. (1) Choosing s, p, r such that $\dot{B}_{p, r}^{s}\left(\mathbb{R}^{n}\right)$ is a Banach space, then we have

$$
\left\|T_{u} v\right\|_{\mathcal{L}^{q / 2}\left(I ; \dot{B}_{p, r}^{s}\right)} \leq\|u\|_{L^{q}\left(I ; L^{\infty}\right)}\|v\|_{\mathcal{L}^{q}\left(I ; \dot{B}_{p, r}^{s}\right)} .
$$

(2) Let $s_{1}<0$ and $\frac{1}{r}=\frac{1}{r_{1}}+\frac{1}{r_{2}}$, and choosing $s_{2}, p, r_{2}$ such that $\dot{B}_{p, r_{2}}^{s_{2}}\left(\mathbb{R}^{n}\right)$ is a Banach space. Then

$$
\left\|T_{u} v\right\|_{\mathcal{L}^{q / 2}\left(I ; \dot{B}_{p, r}^{\left.s_{1}+s_{2}\right)}\right.} \leq C\|u\|_{\mathcal{L}^{q}\left(I ; \dot{B}_{\infty, r_{1}}^{s_{1}}\right)}\|v\|_{\mathcal{L}^{q}\left(I ; \dot{B}_{p, r_{2}}^{s_{2}}\right)} .
$$

According to the definition of the space-time mixed Besov space, a straightforward calculation shows the estimate of the remainder of the paraproduct.

LeMmA 3.2. Let $s_{1}, s_{2} \in \mathbb{R}, 1 \leq p_{1}, p_{2}, p, r_{1}, r_{2}, r \leq \infty$ and $2 \leq q \leq \infty$ such that

$$
\frac{1}{p}=\frac{1}{p_{1}}+\frac{1}{p_{2}}, \quad \frac{1}{r}=\frac{1}{r_{1}}+\frac{1}{r_{2}}
$$


and $\mathcal{L}^{q}\left(I ; \dot{B}_{p_{1}, r_{1}}^{s_{1}}\right), \mathcal{L}^{q}\left(I ; \dot{B}_{p_{2}, r_{2}}^{s_{2}}\right)$ and $\mathcal{L}^{q / 2}\left(I ; \dot{B}_{p, r}^{s_{1}+s_{2}}\right)$ are Banach spaces. Assume $0<s_{1}+$ $s_{2}<\frac{n}{p}$, then

$$
\|R(u, v)\|_{\mathcal{L}^{q / 2}\left(I ; \dot{B}_{p, r}^{s_{1}+s_{2}}\right)} \leq C\|u\|_{\mathcal{L}^{q}\left(I ; \dot{B}_{p_{1}, r_{1}}^{s_{1}}\right)}\|v\|_{\mathcal{L}^{q}\left(I ; \dot{B}_{p_{2}, r_{2}}^{s_{2}}\right)}
$$

Moreover, if $s_{1}+s_{2}=0$ and $\frac{1}{r_{1}}+\frac{1}{r_{2}}=1$, then one has

$$
\|R(u, v)\|_{\mathcal{L}^{q / 2}\left(I ; \dot{B}_{p, \infty}^{0}\right)} \leq C\|u\|_{\mathcal{L}^{q}\left(I ; \dot{B}_{p_{1}, r_{1}}^{s_{1}}\right)}\|v\|_{\mathcal{L}^{q}\left(I ; \dot{B}_{p_{2}, r_{2}}^{s_{2}}\right)} .
$$

If $s_{1}+s_{2}=\frac{n}{p}$ and $r=1$, then

$$
\|R(u, v)\|_{\mathcal{L}^{q / 2}\left(I ; \dot{B}_{p, 1}^{n / p}\right)} \leq C\|u\|_{\mathcal{L}^{q}\left(I ; \dot{B}_{p_{1}, r_{1}}^{s_{1}}\right)}\|v\|_{\mathcal{L}^{q}\left(I ; \dot{B}_{p_{2}, r_{2}}^{s_{2}}\right)} .
$$

We choose $p_{1}=r_{1}=\infty, s_{1}=0$ in Lemma 3.2, noting the fact $L^{\infty} \hookrightarrow \dot{B}_{\infty, \infty}^{0}$ and $\mathcal{L}^{q}\left(I ; \dot{B}_{p, 1}^{n / p}\right) \hookrightarrow L^{q}\left(I ; \dot{B}_{p, 1}^{n / p}\right) \hookrightarrow L^{q}\left(I ; L^{\infty}\right)$ for $q \geq 1$, and combining with Lemma 3.1 we can derive the following results:

COROLlary 3.1. Let $s$ be a real number such that $s<\frac{n}{p}, q \geq 2$ and $1 \leq p, r \leq \infty$. Then we have

$$
\|u v\|_{\mathcal{L}^{q / 2}\left(I ; \dot{B}_{p, r}^{s}\right)} \leq C\|u\|_{L^{q}\left(I ; L^{\infty}\right)}\|v\|_{\mathcal{L}^{q}\left(I ; \dot{B}_{p, r}^{s}\right)}+\|u\|_{\mathcal{L}^{q}\left(I ; \dot{B}_{p, r}^{s}\right)}\|v\|_{L^{q}\left(I ; L^{\infty}\right)},
$$

and

$$
\|u v\|_{\mathcal{L}^{q / 2}\left(I ; \dot{B}_{p, 1}^{n / p}\right)} \leq C\|u\|_{\mathcal{L}^{q}\left(I ; \dot{B}_{p, 1}^{n / p}\right)}\|v\|_{\mathcal{L}^{q}\left(I ; \dot{B}_{p, 1}^{n / p}\right)} .
$$

Corollary 3.2. Let $s_{1}, s_{2} \in \mathbb{R}, 1 \leq p_{k}, r_{k} \leq \infty$ and $1 \leq p, r \leq \infty$ be such that

$$
s_{k}<\frac{n}{p_{k}}, \frac{1}{r_{1}}+\frac{1}{r_{2}}=\frac{1}{r}, p \geq \max \left(p_{1}, p_{2}\right)
$$

for $k=1,2$. If $s_{1}+s_{2}>n\left(\frac{1}{p_{1}}+\frac{1}{p_{2}}-\frac{1}{p}\right)$, then

$$
\|u v\|_{\mathcal{L}^{q / 2}\left(I ; \dot{B}_{p, r}^{s_{1}+s_{2}-n\left(\frac{1}{p_{1}}+\frac{1}{p}{ }_{2}-\frac{1}{p}\right)}\right)} \leq C\|u\|_{\mathcal{L}^{q}\left(I ; \dot{B}_{p_{1}, r_{1}}^{s_{1}}\right)}\|v\|_{\mathcal{L}^{q}\left(I ; \dot{B}_{p_{2}, r_{2}}^{s_{2}}\right)} .
$$

By means of Proposition 3.1 and Corollary 3.2, the Banach contraction mapping principle immediately implies the global well-posedness of the Cauchy problem (1.1)-(1.4) for small data $\left(u_{0}(x), b_{0}(x)\right) \in \dot{B}_{p, r}^{n / p-1}\left(\mathbb{R}^{n}\right)$ and the local well-posedness for arbitrary data $\left(u_{0}(x), b_{0}(x)\right) \in \dot{B}_{p, r}^{n / p-1}\left(\mathbb{R}^{n}\right)$ for $n \geq 2$.

TheOREm 3.1. Let $\left(u_{0}, b_{0}\right) \in \dot{B}_{p, r}^{n / p-1}\left(\mathbb{R}^{n}\right), 1 \leq p<\infty, 2<q \leq \infty$ and $\operatorname{div} u_{0}=$ $\operatorname{div} b_{0}=0$.

(i) For $1 \leq r \leq \infty$, there exists $\varepsilon_{0}>0$ such that if $\left\|\left(u_{0}, b_{0}\right)\right\|_{\dot{B}_{p, r}^{n / p-1}}<\varepsilon_{0}$, then (1.1)-(1.4) has a unique solution $(u, b)$ satisfying

$$
(u, b) \in C_{b}\left(\mathbb{R}^{+} ; \dot{B}_{p, r}^{n / p-1}\right) \cap \mathcal{L}^{q}\left(\mathbb{R}^{+} ; \dot{B}_{p, r}^{s_{p}+2 / q}\right),
$$

where $s_{p}=\frac{n}{p}-1>1-\frac{4}{q}$ is a real number.

(ii) For $1 \leq r<\infty$, there exists a time $T$ and a unique local solution to the system (1.1)-(1.4) $(u(x, t), b(x, t))$ such that

$$
(u, b) \in C_{b}\left([0, T] ; \dot{B}_{p, r}^{n / p-1}\right) \cap \mathcal{L}^{q}\left([0, T] ; \dot{B}_{p, r}^{\frac{n}{p}+\frac{2}{q}-1}\right),
$$

where $p, q$ satisfy $\frac{n}{2 p}+\frac{2}{q}>1$. 
4. Serrin's criterion on the Leray-Hopf weak solutions for MHD system. In this section, we present a sketch of the proof of Serrin's criterion on the Leray-Hopf weak solutions for MHD system following the ideas contained in Lemarié's book [21].

TheOREM 4.1. Let $\left(u_{0}, b_{0}\right) \in L^{2}\left(\mathbb{R}^{n}\right)$ with $\nabla \cdot u_{0}(x)=\nabla \cdot b_{0}(x)=0$. Assume that there exists a Leray-Hopf weak solution $(u, b)$ with initial data $\left(u_{0}, b_{0}\right)$ satisfying one of the following space-time integrability conditions:

(i) For some $r \in(n, \infty],(u, b) \in L^{q}\left((0, T) ; L^{r}\left(\mathbb{R}^{n}\right)\right)$, with $\frac{2}{q}=n\left(\frac{1}{n}-\frac{1}{r}\right)$, for some $T>0$.

(ii) $(u, b) \in L^{2}\left([0, T) ; B M O\left(\mathbb{R}^{n}\right)\right)$, for some $T>0$.

(iii) There exists a constant $\delta_{n}>0$ such that $\|(u, b) ; L^{\infty}\left((0, T) ; L^{n}\left(\mathbb{R}^{n}\right) \|<\delta_{n}\right.$.

Then $(u, b)$ is a unique Leray-Hopf solution associated with initial data $\left(u_{0}, b_{0}\right)$ on $(0, T)$. Moreover, $(u(t), b(t)) \in C\left([0, T) ; L^{2}\left(\mathbb{R}^{n}\right)\right)$ and satisfies the energy equality

$$
\begin{aligned}
& \int_{\mathbb{R}^{n}}\left(|u|^{2}+|b|^{2}\right) d x+2 \nu \int_{s}^{t} \int_{\mathbb{R}^{n}}|\nabla \otimes u|^{2} d x d t+2 \eta \int_{0}^{T} \int_{\mathbb{R}^{n}}|\nabla \otimes b|^{2} d x d t \\
= & \int_{\mathbb{R}^{n}}\left(|u(s)|^{2}+|b(s)|^{2}\right) d x, \quad \text { for all } 0 \leq s<t<T .
\end{aligned}
$$

Proof. (iii) is obvious and we omit its proof. Let $(\tilde{u}, \tilde{b})$ be another Leray-Hopf weak solution associated with the initial datum $\left(u_{0}, b_{0}\right)$. By the regularization method, we easily get, by the formal computation

$$
\begin{aligned}
& \partial_{t} \int_{\mathbb{R}^{n}} u \tilde{u} d x+2 \nu \int_{\mathbb{R}^{n}} \nabla \otimes u \cdot \nabla \otimes \tilde{u} d x \\
= & \int_{\mathbb{R}^{n}} u(u \cdot \nabla) \tilde{u} d x-\int_{\mathbb{R}^{n}} u(\tilde{u} \cdot \nabla) \tilde{u} d x+\int_{\mathbb{R}^{n}} \tilde{u}(b \cdot \nabla) b d x+\int_{\mathbb{R}^{n}} u(\tilde{b} \cdot \nabla) \tilde{b} d x,
\end{aligned}
$$

and

$$
\begin{aligned}
& \partial_{t} \int_{\mathbb{R}^{n}} b \tilde{b} d x+2 \eta \int_{\mathbb{R}^{n}} \nabla \otimes b \cdot \nabla \otimes \tilde{b} d x \\
= & \int_{\mathbb{R}^{n}} b(u \cdot \nabla) \tilde{b} d x-\int_{\mathbb{R}^{n}} b(\tilde{u} \cdot \nabla) \tilde{b} d x+\int_{\mathbb{R}^{n}} \tilde{b}(b \cdot \nabla) u d x+\int_{\mathbb{R}^{n}} b(\tilde{b} \cdot \nabla) \tilde{u} d x,
\end{aligned}
$$

which implies that

$$
\begin{aligned}
& \partial_{t} \int_{\mathbb{R}^{n}}(u \tilde{u}+b \tilde{b}) d x+2 \nu \int_{\mathbb{R}^{n}} \nabla \otimes u \cdot \nabla \otimes \tilde{u} d x+2 \eta \int_{\mathbb{R}^{n}} \nabla \otimes b \cdot \nabla \otimes \tilde{b} d x \\
= & \int_{\mathbb{R}^{n}} u((u-\tilde{u}) \cdot \nabla) \tilde{u} d x+\int_{\mathbb{R}^{n}} b((u-\tilde{u}) \cdot \nabla) \tilde{b} d x \\
& +\int_{\mathbb{R}^{n}} \tilde{u}((b-\tilde{b}) \cdot \nabla) b d x+\int_{\mathbb{R}^{n}} \tilde{b}((b-\tilde{b}) \cdot \nabla) u d x .
\end{aligned}
$$

Thanks to the energy inequality for $(u, b)$ and $(\tilde{u}, \tilde{b})$

$$
\begin{aligned}
& \int_{\mathbb{R}^{n}}\left(|u|^{2}+|b|^{2}\right) d x+2 \nu \int_{0}^{T} \int_{\mathbb{R}^{n}}|\nabla \otimes u|^{2} d x d t+2 \eta \int_{0}^{T} \int_{\mathbb{R}^{n}}|\nabla \otimes b|^{2} d x d t \\
\leq & \int_{\mathbb{R}^{n}}\left(\left|u_{0}\right|^{2}+\left|b_{0}\right|^{2}\right) d x,
\end{aligned}
$$


and

$$
\begin{aligned}
& \int_{\mathbb{R}^{n}}\left(|\tilde{u}|^{2}+|\tilde{b}|^{2}\right) d x+2 \nu \int_{0}^{T} \int_{\mathbb{R}^{n}}|\nabla \otimes \tilde{u}|^{2} d x d t+2 \eta \int_{0}^{T} \int_{\mathbb{R}^{n}}|\nabla \otimes \tilde{b}|^{2} d x d t \\
\leq & \int_{\mathbb{R}^{n}}\left(\left|u_{0}\right|^{2}+\left|b_{0}\right|^{2}\right) d x,
\end{aligned}
$$

we easily verify that

$$
\begin{aligned}
& \|u-\tilde{u}\|_{2}^{2}+\|b-\tilde{b}\|_{2}^{2}=\|u\|_{2}^{2}+\|\tilde{u}\|_{2}^{2}+\|b\|_{2}^{2}+\|\tilde{b}\|_{2}^{2}-2 \int_{\mathbb{R}^{n}} u \tilde{u} d x-2 \int_{\mathbb{R}^{n}} b \tilde{b} d x \\
\leq & -2 \nu \int_{0}^{t} \int_{\mathbb{R}^{n}}|\nabla \otimes u-\nabla \otimes \tilde{u}|^{2} d x d t-2 \eta \int_{0}^{t} \int_{\mathbb{R}^{n}}|\nabla \otimes b-\nabla \otimes \tilde{b}|^{2} d x d t \\
& -\int_{0}^{t} \int_{\mathbb{R}^{n}} u((u-\tilde{u}) \cdot \nabla) \tilde{u} d x d t-\int_{0}^{t} \int_{\mathbb{R}^{n}} b((u-\tilde{u}) \cdot \nabla) \tilde{b} d x d t \\
& +\int_{0}^{t} \int_{\mathbb{R}^{n}}^{t} b((b-\tilde{b}) \cdot \nabla) \tilde{u} d x+\int_{0}^{t} \int_{\mathbb{R}^{n}} u((b-\tilde{b}) \cdot \nabla) \tilde{b} d x d t \\
= & -2 \nu \int_{0}^{t} \int_{\mathbb{R}^{n}}|\nabla \otimes(u-\tilde{u})|^{2} d x d t-2 \eta \int_{0}^{t} \int_{\mathbb{R}^{n}}|\nabla \otimes(b-\tilde{b})|^{2} d x d t \\
& -\int_{0}^{t} \int_{\mathbb{R}^{n}} u((u-\tilde{u}) \cdot \nabla)(\tilde{u}-u) d x d t-\int_{0}^{t} \int_{\mathbb{R}^{n}} b((u-\tilde{u}) \cdot \nabla)(\tilde{b}-b) d x d t \\
& +\int_{0}^{t} \int_{\mathbb{R}^{n}} b((b-\tilde{b}) \cdot \nabla)(\tilde{u}-u) d x d t+\int_{0}^{t} \int_{\mathbb{R}^{n}} u((b-\tilde{b}) \cdot \nabla)(\tilde{b}-b) d x d t \\
= & -2 \nu \int_{0}^{t} \int_{\mathbb{R}^{n}}|\nabla \otimes(u-\tilde{u})|^{2} d x d t-2 \eta \int_{0}^{t} \int_{\mathbb{R}^{n}}|\nabla \otimes(b-\tilde{b})|^{2} d x d t \\
& +I_{1}+I_{2}+I_{3}+I_{4},
\end{aligned}
$$

where we used the fact that

$$
\int_{0}^{t} \int_{\mathbb{R}^{n}} u((u-\tilde{u}) \cdot \nabla) u d x=0, \quad \int_{0}^{t} \int_{\mathbb{R}^{n}} b((u-\tilde{u}) \cdot \nabla) b d x=0 .
$$

Let $\theta=\frac{n}{r}<1$, then

$$
\frac{1}{\varrho}=\frac{r-2}{2 r}=\frac{1}{2}-\frac{\theta}{n}, \quad \frac{q-2}{2 q}=\frac{n}{2 r}=\frac{\theta}{2} .
$$

We apply the Hölder inequality with exponents $r, 2, \varrho$ with respect to $x \in \mathbb{R}^{n}$ and $q, 2$, $\frac{2}{\theta}$ with respect to $t$, respectively, the interpolation inequality and the Young inequality to obtain

$$
\begin{aligned}
\left|I_{1}\right| & \leq\left(\int_{0}^{t}\|u\|_{r}^{q} d t\right)^{\frac{1}{q}}\left(\int_{0}^{t} \int_{\mathbb{R}^{n}}|\nabla \otimes(u-\tilde{u})|^{2} d x d t\right)^{\frac{1}{2}}\left(\int_{0}^{t}\left(\int_{\mathbb{R}^{n}}|\tilde{u}-u|^{\varrho} d x\right)^{\frac{2}{\varrho \theta}} d t\right)^{\frac{\theta}{2}} \\
& \leq C_{\theta}\|u\|_{L^{q}\left(I ; L^{r}\left(\mathbb{R}^{n}\right)\right)}\|\nabla \otimes(u-\tilde{u})\|_{L^{2}\left(I \times \mathbb{R}^{n}\right)}^{1+\theta} \sup _{t \in I}\|\tilde{u}-u\|_{L^{2}\left(\mathbb{R}^{n}\right)}^{1-\theta} \\
& \leq C_{\theta}\left(\int_{0}^{t}\|u\|_{r}^{q} d t\right)^{\frac{1}{q}}\left(\frac{1+\theta}{2}\|\nabla \otimes(u-\tilde{u})\|_{L^{2}\left(I \times \mathbb{R}^{n}\right)}^{2}+\frac{1-\theta}{2} \sup _{t \in I}\|u-\tilde{u}\|_{2}^{2}\right),
\end{aligned}
$$


and

$$
\begin{aligned}
\left|I_{2}\right| \leq & \left(\int_{0}^{t}\|b\|_{r}^{q} d t\right)^{\frac{1}{q}}\left(\int_{0}^{t} \int_{\mathbb{R}^{n}}|\nabla \otimes(b-\tilde{b})|^{2} d x d t\right)^{\frac{1}{2}}\left(\int_{0}^{t}\left(\int_{\mathbb{R}^{n}}|\tilde{u}-u|^{\varrho} d x\right)^{\frac{2}{\varrho \theta}} d t\right)^{\frac{\theta}{2}} \\
\leq & C_{\theta}\|b\|_{L^{q}\left(I ; L^{r}\left(\mathbb{R}^{n}\right)\right)}\|\nabla \otimes(b-\tilde{b})\|_{L^{2}\left(I \times \mathbb{R}^{n}\right)}\|\nabla \otimes(u-\tilde{u})\|_{L^{2}\left(I \times \mathbb{R}^{n}\right)}^{\theta} \sup _{t \in I}\|\tilde{u}-u\|_{L^{2}\left(\mathbb{R}^{n}\right)}^{1-\theta} \\
\leq & C_{\theta}\left(\int_{0}^{t}\|b\|_{r}^{q} d t\right)^{\frac{1}{q}}\left(\frac{1+\theta}{2}\|\nabla \otimes(b-\tilde{b})\|_{L^{2}\left(I \times \mathbb{R}^{n}\right)}^{\frac{2}{1+\theta}}\|\nabla \otimes(u-\tilde{u})\|_{L^{2}\left(I \times \mathbb{R}^{n}\right)}^{\frac{2 \theta}{1+\theta}}\right. \\
& \left.+\frac{1-\theta}{2} \sup _{t \in I}\|u-\tilde{u}\|_{2}^{2}\right) \\
\leq & C_{\theta}\left(\int_{0}^{t}\|b\|_{r}^{q} d t\right)^{\frac{1}{q}}\left(\frac{1}{2}\|\nabla \otimes(b-\tilde{b})\|_{L^{2}\left(I \times \mathbb{R}^{n}\right)}^{2}+\frac{\theta}{2}\|\nabla \otimes(u-\tilde{u})\|_{L^{2}\left(I \times \mathbb{R}^{n}\right)}^{2}\right. \\
& \left.+\frac{1-\theta}{2} \sup _{t \in I}\|u-\tilde{u}\|_{2}^{2}\right) .
\end{aligned}
$$

In exactly the same way as leading to (4.4) and (4.5), we have

$$
\begin{aligned}
\left|I_{4}\right| \leq & \left(\int_{0}^{t}\|u\|_{r}^{q} d t\right)^{\frac{1}{q}}\left(\frac{1+\theta}{2} \int_{0}^{t} \int_{\mathbb{R}^{n}}|\nabla \otimes(b-\tilde{b})|^{2} d x d t\right. \\
& \left.+\frac{1-\theta}{2} \sup _{t \in I}\|b-\tilde{b}\|_{2}^{2}\right),
\end{aligned}
$$

and

$$
\begin{aligned}
\left|I_{3}\right| \leq & C_{\theta}\left(\int_{0}^{t}\|b\|_{r}^{q} d t\right)^{\frac{1}{q}}\left(\frac{1}{2}\|\nabla \otimes(u-\tilde{u})\|_{L^{2}\left(I \times \mathbb{R}^{n}\right)}^{2}+\frac{\theta}{2} \mid \nabla \otimes(b-\tilde{b}) \|_{L^{2}\left(I \times \mathbb{R}^{n}\right)}^{2}\right. \\
& \left.+\frac{1-\theta}{2} \sup _{t \in I}\|b-\tilde{b}\|_{2}^{2}\right) .
\end{aligned}
$$

We collect (4.3)-(4.7) to get

$$
\begin{aligned}
& \sup _{t \in I}\|u-\tilde{u}\|_{2}^{2}+\sup _{t \in I}\|b-\tilde{b}\|_{2}^{2} \\
\leq & -2 \nu\|\nabla \otimes(u-\tilde{u})\|_{L^{2}\left(I \times \mathbb{R}^{n}\right)}^{2}-2 \eta\|\nabla \otimes(b-\tilde{b})\|_{L^{2}\left(I \times \mathbb{R}^{n}\right)}^{2} \\
& +C_{\theta}\left[\left(\int_{0}^{t}\|u\|_{r}^{q} d t\right)^{\frac{1}{q}}+\left(\int_{0}^{t}\|b\|_{r}^{q} d t\right)^{\frac{1}{q}}\right] \\
& \times\left[\frac{1+\theta}{2}\left(\|\nabla \otimes(u-\tilde{u})\|_{L^{2}\left(I \times \mathbb{R}^{n}\right)}^{2}+\|\nabla \otimes(b-\tilde{b})\|_{L^{2}\left(I \times \mathbb{R}^{n}\right)}^{2}\right)\right. \\
& \left.+\frac{1-\theta}{2}\left(\sup _{t \in I}\|u-\tilde{u}\|_{2}^{2}+\sup _{t \in I}\|b-\tilde{b}\|_{2}^{2}\right)\right] .
\end{aligned}
$$

Choosing a suitable small $t>0$ such that

$$
\frac{1+\theta}{2} C_{\theta}\left[\left(\int_{0}^{t}\|u\|_{r}^{q} d t\right)^{\frac{1}{q}}+\left(\int_{0}^{t}\|b\|_{r}^{q} d t\right)^{\frac{1}{q}}\right]<\min (2 \nu, 2 \eta, 1)
$$

this gives

$$
(u, b)=(\tilde{u}, \tilde{b}), \quad \text { in } \quad I=[0, t] .
$$


One repeats the above process to get $(u, b)=(\tilde{u}, \tilde{b})$ on $[0, T)$ provided that $n<r \leq \infty$. When $q=\infty, r=n$, we have to assume that

$$
C_{1}\left[\sup _{t \in I}\|u\|_{n}+\sup _{t \in I}\|b\|_{n}\right]<1
$$

to get the uniqueness. For any scale function $g \in \dot{H}^{1}\left(\mathbb{R}^{n}\right)$ and vector-valued function $f \in L^{n}\left(\mathbb{R}^{n}\right)$ with $\nabla \cdot f=0$, the well-known div-curl theorem implies

$$
\|f \cdot \nabla g\|_{\mathcal{H}^{1}} \leq C\|f\|_{2}\|\nabla g\|_{2},
$$

where $\mathcal{H}^{1}$ denotes the Hardy space. This gives the following estimates:

$$
\begin{aligned}
\left|I_{1}\right| & \leq\left(\int_{0}^{t}\|u\|_{\mathrm{BMO}}^{2} d t\right)^{\frac{1}{2}}\left(\int_{0}^{t}\|((\tilde{u}-u) \cdot \nabla)(u-\tilde{u})\|_{\mathcal{H}^{1}}^{2} d t\right)^{\frac{1}{2}} \\
& \leq C_{0}\|u\|_{L^{2}(I ; \mathrm{BMO})}\|\nabla \otimes(u-\tilde{u})\|_{L^{2}\left(I \times \mathbb{R}^{n}\right)} \sup _{t \in I}\|\tilde{u}-u\|_{L^{2}} \\
& \leq \frac{C_{0}}{2}\left(\int_{0}^{t}\|u\|_{\mathrm{BMO}}^{2} d t\right)^{\frac{1}{2}}\left(\|\nabla \otimes(u-\tilde{u})\|_{L^{2}\left(I \times \mathbb{R}^{n}\right)}^{2}+\sup _{t \in I}\|u-\tilde{u}\|_{2}^{2}\right), \\
\left|I_{2}\right| & \leq \frac{C_{0}}{2}\left(\int_{0}^{t}\|b\|_{\mathrm{BMO}}^{2} d t\right)^{\frac{1}{2}}\left(\|\nabla \otimes(b-\tilde{b})\|_{L^{2}\left(I \times \mathbb{R}^{n}\right)}^{2}+\sup _{t \in I}\|u-\tilde{u}\|_{2}^{2}\right), \\
\left|I_{3}\right| & \leq \frac{C_{0}}{2}\left(\int_{0}^{t}\|b\|_{\mathrm{BMO}}^{2} d t\right)^{\frac{1}{2}}\left(\|\nabla \otimes(u-\tilde{u})\|_{L^{2}\left(I \times \mathbb{R}^{n}\right)}^{2}+\sup _{t \in I}\|b-\tilde{b}\|_{2}^{2}\right), \\
\left|I_{4}\right| & \leq \frac{C_{0}}{2}\left(\int_{0}^{t}\|u\|_{\mathrm{BMO}}^{2} d t\right)^{\frac{1}{2}}\left(\|\nabla \otimes(b-\tilde{b})\|_{L^{2}\left(I \times \mathbb{R}^{n}\right)}^{2}+\sup _{t \in I}\|b-\tilde{b}\|_{2}^{2}\right),
\end{aligned}
$$

which implies

$$
\begin{aligned}
& \sup _{t \in I}\|u-\tilde{u}\|_{2}^{2}+\sup _{t \in I}\|b-\tilde{b}\|_{2}^{2} \\
& \leq-2 \nu\|\nabla \otimes(u-\tilde{u})\|_{L^{2}\left(I \times \mathbb{R}^{n}\right)}^{2}-2 \eta\|\nabla \otimes(b-\tilde{b})\|_{L^{2}\left(I \times \mathbb{R}^{n}\right)}^{2} \\
& +\frac{C_{0}}{2}\left[\left(\int_{0}^{t}\|u\|_{\mathrm{BMO}}^{2} d t\right)^{\frac{1}{2}}+\left(\int_{0}^{t}\|b\|_{\mathrm{BMO}}^{2} d t\right)^{\frac{1}{2}}\right] \times \\
& \quad \times\left[\|\nabla \otimes(u-\tilde{u})\|_{L^{2}\left(I \times \mathbb{R}^{n}\right)}^{2}+\|\nabla \otimes(b-\tilde{b})\|_{L^{2}\left(I \times \mathbb{R}^{n}\right)}^{2}\right. \\
& \left.+\sup _{t \in I}\|u-\tilde{u}\|_{2}^{2}+\sup _{t \in I}\|b-\tilde{b}\|_{2}^{2}\right] .
\end{aligned}
$$

This gives the uniqueness of the Leray-Hopf weak solutions associated to the initial data $\left(u_{0}(x), b_{0}(x)\right)$ in the case of $(u, b) \in L^{2}\left([0, T) ; \operatorname{BMO}\left(\mathbb{R}^{n}\right)\right)$.

REMARK 4.1. (i) Similarly to the case of the Navier-Stokes equations, Theorem 4.1 gives $(u, b) \in C^{\infty}\left((0, T) \times \mathbb{R}^{n}\right)$ by the equivalence between uniqueness and regularity.

(ii) When $n=2, \dot{H}^{1}\left(\mathbb{R}^{2}\right) \hookrightarrow \operatorname{BMO}\left(\mathbb{R}^{2}\right)$. Hence, the Leray-Hopf weak solution associated with the initial data $\left(u_{0}(x), b_{0}(x)\right)$ is unique and regular.

(iii) If the Leray-Hopf weak solution $(u, b) \in C\left([0, T), L^{n}\right)$, then it is unique and regular. 
(iv) When $n \geq 4$, the Leray-Hopf weak solution is unique and regular, provided that $(u, b) \in L^{\infty}\left([0, T), L^{n}\right)$. The proof is similar to the case of the Navier-Stokes equations, see Chap. 28 in [21].

(v) When $n=3$, is the Leray-Hopf weak solution unique and regular, provided that $(u, b) \in L^{\infty}\left([0, T), L^{3}\right)$ ? This is also an open problem.

5. Uniqueness and regularity criterion based on fluid vorticity and electrical current in BMO and Besov spaces. Similarly to the case of the Navier-Stokes equations $[2,4,19,31]$, we establish the uniqueness or regularity criterion for the MHD system (1.1)-(1.4).

TheOREM 5.1. Let $\left(u_{0}(x), b_{0}(x)\right) \in L^{2}\left(\mathbb{R}^{n}\right)$ with $\nabla \cdot u_{0}=\nabla \cdot b_{0}=0$, and suppose that $(u, b) \in L^{\infty}\left((0, T) ; L^{2}\left(\mathbb{R}^{n}\right)\right) \cap L^{2}\left((0, T) ; \dot{H}^{1}\left(\mathbb{R}^{n}\right)\right)$ is a Leray-Hopf weak solution which satisfies the energy inequality

$$
\begin{aligned}
& \int_{\mathbb{R}^{n}}\left(|u(t)|^{2}+|b(t)|^{2}\right) d x+2 \nu \int_{0}^{t} \int_{\mathbb{R}^{n}}|\nabla \otimes u|^{2} d x d t+2 \eta \int_{0}^{t} \int_{\mathbb{R}^{n}}|\nabla \otimes b|^{2} d x d t \\
\leq & \int_{\mathbb{R}^{n}}\left(|u(s)|^{2}+|b(s)|^{2}\right) d x, \quad \text { for } \text { a.e. } t \geq 0 \quad \text { and } \quad s \leq t .
\end{aligned}
$$

(i) Assume that the solution $(u, b)$ satisfies

$$
\left(\int_{0}^{T}\|\omega\|_{r}^{q} d t\right)^{\frac{1}{q}}<\infty, \quad\left(\int_{0}^{T}\|j\|_{r}^{q} d t\right)^{\frac{1}{q}}<\infty,
$$

where $(q, r) \in \tilde{\Lambda}_{1}$ with $r \neq \frac{n}{2}$. Then the solution $(u, b)$ is smooth on $(0, T]$.

(ii) More generally for the endpoint case, if we assume that the solution $(u, b)$ satisfies

$$
\int_{0}^{T}\|\omega\|_{\dot{B}_{\infty, \infty}^{0}} d t<\infty, \quad \int_{0}^{T}\|j\|_{\dot{B}_{\infty, \infty}^{0}} d t<\infty,
$$

then the solution $(u, b)$ is smooth on $(0, T]$.

REMARK 5.1. (i) Since

$$
L^{2}\left([0, T) ; L^{2}\left(\mathbb{R}^{n}\right)\right) \cap L^{\infty}\left([0, T) ; \dot{H}^{1}\left(\mathbb{R}^{n}\right)\right) \hookrightarrow L^{2}\left([0, T) ; L^{4}\left(\mathbb{R}^{n}\right)\right), \quad n \leq 4,
$$

one easily verifies that each Leray-Hopf weak solution satisfies the strong type energy inequality (5.1), see [21] for the proof.

(ii) By means of the boundedness of singular integral operators in Besov spaces and

$$
\nabla u=(-\triangle)^{-1} \nabla \operatorname{rot} \omega, \quad \nabla b=(-\triangle)^{-1} \nabla \operatorname{rot} j
$$

one easily shows that (5.2) and (5.3) are equivalent to

$$
\left(\int_{0}^{T}\|\nabla u\|_{r}^{q} d t\right)^{\frac{1}{q}}<\infty, \quad\left(\int_{0}^{T}\|\nabla b\|_{r}^{q} d t\right)^{\frac{1}{q}}<\infty
$$

and

$$
\int_{0}^{T}\|\nabla u\|_{\dot{B}_{\infty, \infty}^{0}} d t<\infty, \quad \int_{0}^{T}\|\nabla b\|_{\dot{B}_{\infty, \infty}^{0}} d t<\infty
$$

respectively. 
(iii) Let $(u, b) \in L^{\infty}\left((0, T) \times \mathbb{R}^{n}\right)$ be a Leray-Hopf weak solution of MHD equations, then $(u, b) \in C^{\infty}\left((0, T) \times \mathbb{R}^{n}\right)$. The proof is similar to that for the Navier-Stokes equations, see $[21]$ for details.

Proof of Theorem 5.1. For any $(q, r) \in \Lambda_{1}$ with $\frac{n}{2}<r \leq \infty$, we choose

$$
\frac{1}{r_{1}}=\frac{1}{r}-\frac{1}{n}
$$

so that $\left(q, r_{1}\right) \in \Lambda$. This fact and the Sobolev embedding theorem imply that $u \in$ $L^{q}\left(I ; L^{r_{1}}\right)$ and so we obtain the $u(t, x) \in C^{\infty}\left((0, T] \times \mathbb{R}^{n}\right)$ by the Serrin type regularity criterion, see Section 4.

Formal computations yield

$$
\begin{aligned}
& \frac{1}{n} \frac{d}{d t} \int_{\mathbb{R}^{n}}\left[|u|^{n}+|b|^{n}\right] d x+\frac{4(n-1)}{n^{2}}\left[\nu \int_{\mathbb{R}^{n}}\left|\nabla\left(|u|^{\frac{n}{2}}\right)\right|^{2} d x+\eta \int_{\mathbb{R}^{n}}\left|\nabla\left(|b|^{\frac{n}{2}}\right)\right|^{2} d x\right] \\
= & \int_{\mathbb{R}^{n}}(b \cdot \nabla) b \cdot|u|^{n-2} u d x+\int_{\mathbb{R}^{n}}(b \cdot \nabla) u \cdot|b|^{n-2} b d x-\int_{\mathbb{R}^{n}}(u \cdot \nabla \Pi)|u|^{n-2} d x \\
= & I_{1}+I_{2}+I_{3} .
\end{aligned}
$$

We apply the Hölder inequality with exponents $\left(\frac{n r}{2 r-n}, \frac{n}{n-2}, r\right)$, and the interpolation theorem to obtain

$$
\begin{aligned}
\left|I_{1}\right| & \leq\|b\|_{\frac{2 n r}{2 r-n}}\|u\|_{\frac{2 n r}{2 r-n}}\|u\|_{n}^{n-2}\|\nabla b\|_{r} \\
& \leq C\|b\|_{n^{\frac{2(r-n)}{2 r-n}}}^{n}\|u\|_{n}^{n-2+\frac{2(r-n)}{2 r-n}}\|b\|_{2 r}^{\frac{n}{2 r-n}}\|u\|_{2 r}^{\frac{n}{2 r-n}}\|\nabla b\|_{r} \\
& \leq C\|b\|_{n}^{\frac{4(2 r-3 n}{2(2 r-n)}}\|u\|_{n}^{n-2+\frac{4 r-3 n}{2(2 r-n)}}\|\nabla b\|_{r}^{\frac{4 r-n}{2(2 r-n)}}\|\nabla u\|_{r}^{\frac{n}{2(2 r-n)}} \\
& \leq C\left(\|u\|_{n}+\|b\|_{n}\right)^{n\left(1-\frac{1}{2 r-n}\right)}\left(\|\nabla u\|_{r}^{\frac{2 r}{2 r-n}}+\|\nabla b\|_{r}^{\frac{2 r}{2 r-n}}\right) .
\end{aligned}
$$

In the same way as in the proof of $(5.5)$, one gets

$$
\left|I_{2}\right| \leq C\left(\|u\|_{n}+\|b\|_{n}\right)^{n\left(1-\frac{1}{2 r-n}\right)}\left(\|\nabla u\|_{r}^{\frac{2 r}{2 r-n}}+\|\nabla b\|_{r}^{\frac{2 r}{2 r-n}}\right) .
$$

For the estimate of $I_{3}$, we have to apply the Calderón-Zygmund inequality, Hölder inequality and the interpolation theorem to obtain

$$
\begin{aligned}
\left|I_{3}\right| & =\left.\left|-(n-2) \int_{\mathbb{R}^{n}} \Pi \nabla u\right| u\right|^{n-2} d x \mid \leq(n-2)\|\nabla u\|_{r}\|u\|_{n}^{n-2}\|\Pi\|_{\frac{r n}{2 r-n}} \\
& \leq C \mid \nabla u\left\|_{r}\right\| u \|_{n}^{n-2}\left[\|u\|_{\frac{2 r n}{2 r-n}}^{2}+\|b\|_{\frac{2 r n}{2 r-n}}^{2}\right] \\
& \leq C\left(\|u\|_{n}+\|b\|_{n}\right)^{n\left(1-\frac{1}{2 r-n}\right)}\left(\|\nabla u\|_{r}^{\frac{2 r}{2 r-n}}+\|\nabla b\|_{r}^{\frac{2 r}{2 r-n}}\right) .
\end{aligned}
$$

Let $\delta=\frac{1}{2 r-n}$, putting (5.5)-(5.7) into (5.4) and integrating both sides of (5.4), we have

$$
\begin{gathered}
\|u\|_{n}^{n}+\|b\|_{n}^{n}+\frac{4(n-1)}{n}\left[\nu \int_{0}^{T} \int_{\mathbb{R}^{n}}\left|\nabla\left(|u|^{\frac{n}{2}}\right)\right|^{2} d x+\eta \int_{0}^{T} \int_{\mathbb{R}^{n}}\left|\nabla\left(|b|^{\frac{n}{2}}\right)\right|^{2} d x\right] \\
\leq\left\|u_{0}\right\|_{n}^{n}+\left\|b_{0}\right\|_{n}^{n}+C \int_{0}^{T}\left(\|u\|_{n}+\|b\|_{n}\right)^{n(1-\delta)}\left(\|\nabla u\|_{r}^{\frac{2 r}{2 r-n}}+\|\nabla b\|_{r}^{\frac{2 r}{2 r-n}}\right) d t
\end{gathered}
$$




$$
\begin{aligned}
\leq & \left\|u_{0}\right\|_{n}^{n}+\left\|b_{0}\right\|_{n}^{n}+\frac{1}{2} \sup _{t \in[0, T]}\|u\|_{n}^{n}+\frac{1}{2} \sup _{t \in[0, T]}\|b\|_{n}^{n} \\
& +C\left[\|\nabla u\|_{L^{q}\left(I ; L^{r}\left(\mathbb{R}^{n}\right)\right)}^{\frac{q}{\delta}}+\|\nabla b\|_{L^{q}\left(I ; L^{r}\left(\mathbb{R}^{n}\right)\right)}^{\frac{q}{\delta}}\right], \quad q=\frac{2 r}{2 r-n}
\end{aligned}
$$

by the Young inequality. Taking the $L^{\infty}$-norm with respect to $t$ of both sides of the above inequality, we arrive at

$$
\begin{aligned}
& \|u\|_{L^{\infty}\left(I ; L^{n}\left(\mathbb{R}^{n}\right)\right)}^{n}+\|b\|_{L^{\infty}\left(I ; L^{n}\left(\mathbb{R}^{n}\right)\right)}^{n} \\
& +\frac{4(n-1)}{n}\left[\nu \int_{0}^{T} \int_{\mathbb{R}^{n}}\left|\nabla\left(|u|^{\frac{n}{2}}\right)\right|^{2} d x+\eta \int_{0}^{T} \int_{\mathbb{R}^{n}}\left|\nabla\left(|b|^{\frac{n}{2}}\right)\right|^{2} d x\right] \\
\leq & 2\left\|u_{0}\right\|_{n}^{n}+2 \|\left. b_{0}\right|_{n} ^{n}+C\left[\|\nabla u\|_{L^{q}\left(I ; L^{r}\left(\mathbb{R}^{n}\right)\right.}^{\frac{q}{\delta}}+\|\nabla b\|_{L^{q}\left(I ; L^{r}\left(\mathbb{R}^{n}\right)\right.}^{\frac{q}{\delta}}\right],
\end{aligned}
$$

where $\frac{2}{q}=n\left(\frac{2}{n}-\frac{1}{r}\right)$, i.e. $(q, r) \in \tilde{\Lambda}_{1}$. Hence

$$
(u, b) \in L^{\infty}\left(I ; L^{n}\left(\mathbb{R}^{n}\right)\right)
$$

by the conditions in Theorem 5.1. In particular, we have for $r=\infty$ or $\delta=0$

$$
\begin{aligned}
& \|u\|_{n}^{n}+\|b\|_{n}^{n}+\frac{4(n-1)}{n}\left[\nu \int_{0}^{T} \int_{\mathbb{R}^{n}}\left|\nabla\left(|u|^{\frac{n}{2}}\right)\right|^{2} d x+\eta \int_{0}^{T} \int_{\mathbb{R}^{n}}\left|\nabla\left(|b|^{\frac{n}{2}}\right)\right|^{2} d x\right] \\
\leq & \left\|u_{0}\right\|_{n}^{n}+\|\left. b_{0}\right|_{n} ^{n}+C \int_{0}^{T}\left(\|u\|_{n}+\|\left. b\right|_{n}\right)^{n}\left(\|\nabla u\|_{\infty}+\|\nabla b\|_{\infty}\right) d t .
\end{aligned}
$$

so we also get (5.9) by the Gronwall inequality.

On the other hand, one easily verifies that (5.9) and (5.10) imply

$$
\nu \int_{0}^{T} \int_{\mathbb{R}^{n}}\left|\nabla\left(|u|^{\frac{n}{2}}\right)\right|^{2} d x+\eta \int_{0}^{T} \int_{\mathbb{R}^{n}}\left|\nabla\left(|b|^{\frac{n}{2}}\right)\right|^{2} d x<\infty .
$$

Thanks to the Sobolev embedding theorem, we have

$$
u^{\frac{n}{2}}, b^{\frac{n}{2}} \in L^{2}\left((0, T) ; L^{\frac{2 n}{n-2}}\left(\mathbb{R}^{n}\right)\right) \text { i.e. } \quad(u, b) \in L^{n}\left([0, T) ; L^{\frac{n^{2}}{n-2}}\left(\mathbb{R}^{n}\right)\right) .
$$

We now apply the interpolation theorem to (5.9) and (5.12) to obtain

$$
(u, b) \in L^{q_{1}}\left(I ; L^{r_{1}}\left(\mathbb{R}^{n}\right)\right), \quad\left(q_{1}, r_{1}\right) \in \Lambda,
$$

where

$$
\frac{1}{q_{1}}=\frac{\theta}{\infty}+\frac{1-\theta}{n}, \quad \frac{1}{r_{1}}=\frac{\theta}{n}+\frac{(n-2)(1-\theta)}{n^{2}}, \quad 0<\theta<1 .
$$

Now we are in a position to prove (i) in Theorem 5.1. By the well-known existence theory, for each $\varepsilon>0$, there exists $0<t_{0}<\varepsilon$ such that

$$
\left(u\left(t_{0}\right), b\left(t_{0}\right)\right) \in L^{r}\left(\mathbb{R}^{n}\right), \quad n<r \leq \frac{n^{2}}{n-2} .
$$

Thus, we solve the MHD equations with the initial datum $\left(u\left(t_{0}\right), b\left(t_{0}\right)\right)$ and get the existence of a smooth solution such that $(u(t), b(t)) \in C\left(\left[t_{0}, T\right) ; L^{r}\left(\mathbb{R}^{n}\right)\right)$ by Theorem 2.1 and Theorem 2.2, where $I=[0, T)$ is the largest existence interval. If $T<\infty$, then for any $r>n$,

$$
\|b(\tau)\|_{r}+\|u(\tau)\|_{r} \geq \frac{C}{(T-\tau)^{\frac{r-n}{2 r}}} .
$$


Now we restrict $r \leq \frac{n^{2}}{n-2}$, and construct $q$ such that $(q, r, n)$ is an admissible triplet, then

$$
\infty=\int_{t_{0}}^{T} \frac{C^{q}}{(T-\tau)^{\frac{q(r-n)}{2 r}}} d \tau \leq \int_{t_{0}}^{T}\left[\|u\|_{r}^{q}+\|b\|_{r}^{q}\right] d \tau<\infty .
$$

This contradicts $(u(t), b(t)) \in L^{q}\left(\left[t_{0}, T\right) ; L^{r}\left(\mathbb{R}^{n}\right)\right)$, and we can extend $(u(t), b(t))$ to $t>T$. Thus, we proved that $(u(t), b(t)) \in C^{\infty}\left((0, T] \times \mathbb{R}^{n}\right)$.

REMARK 5.2. In general, we have to assume that the initial condition $\left(u_{0}(x), b_{0}(x)\right) \in$ $L^{p}\left(\mathbb{R}^{n}\right) \cap L^{2}\left(\mathbb{R}^{n}\right)$ with $p \geq n$ in $\mathbb{R}^{n}$. But, when $n=3$ or $n=4$, there exists a sufficiently small $\eta>0$ such that

$$
(u(\eta), b(\eta)) \in H^{1}\left(\mathbb{R}^{n}\right) \hookrightarrow L^{n}\left(\mathbb{R}^{n}\right),
$$

so we can remove the condition $\left(u_{0}(x), b_{0}(x)\right) \in L^{p}\left(\mathbb{R}^{n}\right), p \geq n$.

To prove the (ii) of Theorem 5.1, we first introduce the so-called logarithmic Sobolev inequality in Besov space which can be proved by the Littlewood-Paley decomposition technique, see [19] for details.

Proposition 5.1 (Logarithmic Sobolev inequality in Besov space).

(i) Let $p, \rho, \varrho \in[1, \infty], q \in[1, \infty), s>\frac{n}{q}$, then

$$
\|f\|_{\infty} \leq C\left(1+\|f\|_{\dot{B}_{p, \rho}^{p}}\left(\log ^{+}\|f\|_{B_{q, \varrho}^{s}}\right)^{1-\frac{1}{\rho}}\right), \quad \forall f \in \dot{B}_{p, \rho}^{\frac{n}{p}} \cap B_{q, \varrho}^{s},
$$

where

$$
\log ^{+} t= \begin{cases}\log t, & t>e \\ 1, & 0 \leq t \leq e\end{cases}
$$

(ii) Let $p, q, \rho, \varrho, \nu \in[1, \infty], \nu \leq \min (\rho, \varrho), \frac{1}{q}=\frac{1}{p}-\frac{s}{n}, 1 \leq r \leq q$ satisfy

$$
\frac{1}{r}-\frac{s_{1}}{n}>\frac{1}{q}>\frac{1}{r}-\frac{s_{2}}{n} \text {. }
$$

Then for each $f \in \dot{B}_{r, \varrho}^{s_{1}} \cap \dot{B}_{r, \varrho}^{s_{2}}$,

$$
\|f\|_{\dot{B}_{q, \nu}^{0}} \leq C\left(1+\|f\|_{\dot{B}_{p, \rho}^{s}} \log ^{+}\left(\|f\|_{\dot{B}_{r, \varrho}^{s_{1}}}+\|f\|_{\dot{B}_{r, \varrho}^{s_{2}}}\right)^{\frac{1}{\nu}-\frac{1}{\rho}}\right) .
$$

We first show that the weak solution satisfying the regularity assumption (5.3) or more general regularity conditions $(u, b) \in L^{q}\left(I ; \dot{B}_{r, \infty}^{0}\right),(q, r) \in \tilde{\Lambda}_{1}$, coincides with the smooth solution constructed from the integral equation in a time interval. By means of Theorem 2.1 or Theorem 2.2, for initial data $\left(\tilde{u}_{0}(x), \tilde{b}_{0}(x)\right) \in L^{n} \cap H^{1}$, there exist $\tilde{T}>0$ and a strong solution $(\tilde{u}(t), \tilde{b}(t))$ of the MHD equations (1.1)-(1.4) or (2.19) such that

$$
(\tilde{u}(t), \tilde{b}(t)) \in C\left([0, \tilde{T}) ; L^{n} \cap H^{1}\right), \quad \tilde{T}=\tilde{T}\left(\left\|\tilde{u}_{0}(x)\right\|_{n},\left\|\tilde{b}_{0}(x)\right\|_{n}\right) .
$$

Moreover, $(u, b)$ satisfies the following regularity property

$$
(\tilde{u}(t), \tilde{b}(t)) \in C\left((0, \tilde{T}) ; H^{s}\right), \quad \forall s \geq 1 .
$$

Secondly, $(u, b) \in L^{2}\left((0, T) ; H^{1}\left(\mathbb{R}^{n}\right)\right)$ together with the regularity condition

$$
\left.\nabla \times u, \nabla \times b \in L^{1}\left((0, T) ; \dot{B}_{\infty, \infty}^{0}\right)\right)
$$

yields

$$
(u(\varepsilon), b(\varepsilon)) \in \dot{B}_{\infty, 1}^{0} \cap H^{1} \hookrightarrow L^{n} \cap H^{1}, \quad \text { a.e. } \quad \varepsilon \in(0, T)
$$


In fact, making use of the interpolation relation $\dot{B}_{t, \rho}^{s}=\left(\dot{B}_{\infty, \infty}^{1}, \dot{B}_{2,2}^{0}\right)_{s}$, where

$$
s=\frac{n}{n+2}, \quad \frac{1}{t}=\frac{1}{\rho}=\frac{1-s}{2},
$$

we show that by Proposition 5.1

$$
\|f\|_{\dot{B}_{\infty, 1}^{0}} \leq C\left(1+\|f\|_{\dot{B}_{\rho, \rho}^{s}} \log ^{+}\left(\|f\|_{\dot{B}_{\infty, \infty}^{s_{1}}}+\|f\|_{\dot{B}_{\infty, \infty}^{s_{2}}}\right)\right),
$$

where $f \in \dot{B}_{\infty, \infty}^{s_{1}} \cap \dot{B}_{\infty, \infty}^{s_{2}}, s_{1}<1-\frac{n}{2}$, and $s_{2}=1$.

Finally, if we solve the MHD equations (1.1)-(1.4) with the initial data $\left(\tilde{u}_{0}(\varepsilon), \tilde{b}_{0}(\varepsilon)\right)=$ $(u(\varepsilon), b(\varepsilon)) \in L^{n} \cap H^{1}$, then there exist $\tilde{T}_{\varepsilon}>0$ and a strong solution $(\tilde{u}(t), \tilde{b}(t))$ on $\left[\varepsilon, \tilde{T}_{\varepsilon}\right)$ such that

$$
(\tilde{u}(t), \tilde{b}(t)) \in C\left(\left[\varepsilon, \tilde{T}_{\varepsilon}\right) ; L^{n} \cap H^{1}\right) \cap L^{q}\left(\left[\varepsilon, \tilde{T}_{\varepsilon}\right) ; L^{r}\left(\mathbb{R}^{n}\right)\right),
$$

where $(q, r, n)$ be any admissible triplet $r>n$. Obviously, $(\tilde{u}(t), \tilde{b}(t))$ is smooth by the Serrin criterion, please refer to Section 4.

Note that the weak solution $(u, b)$ satisfies the energy inequality

$$
\begin{aligned}
& \int_{\mathbb{R}^{n}}\left(|u(t)|^{2}+|b(t)|^{2}\right) d x+2 \nu \int_{\varepsilon}^{t} \int_{\mathbb{R}^{n}}|\nabla \otimes u|^{2} d x d t+2 \eta \int_{\varepsilon}^{t} \int_{\mathbb{R}^{n}}|\nabla \otimes b|^{2} d x d t \\
\leq & \int_{\mathbb{R}^{n}}\left(|u(\varepsilon)|^{2}+|b(\varepsilon)|^{2}\right) d x, \quad \varepsilon \leq t \leq T .
\end{aligned}
$$

Serrin's uniqueness criterion implies that

$$
\begin{gathered}
u \equiv \tilde{u}(t), \quad t \in\left(\varepsilon, \tilde{T}_{\varepsilon}\right), \\
(\tilde{u}(t), \tilde{b}(t)) \in C\left(\left[\varepsilon, \tilde{T}_{\varepsilon}\right) ; L^{n} \cap H^{1}\right) \cap C^{1}\left(\left(\varepsilon, \tilde{T}_{\varepsilon}\right) ; H^{s}\right) \quad \forall s \geq 1 .
\end{gathered}
$$

Therefore $u(t)$ is regular on $\left(\varepsilon, \tilde{T}_{\varepsilon}\right)$. If $T \leq \tilde{T}_{\varepsilon}$, the proof is complete. Otherwise, we continue the above argument until it fails.

Now we only prove the regularity of $(u(t), b(t))$ at $t=T$ using a similar argument as in [19] for the case of Navier-Stokes equations. Choosing a suitable multiindex $|\alpha|>\frac{n}{2}+1$, one gets

$$
\left\{\begin{array}{l}
\partial_{t} \partial^{\alpha} u+\partial^{\alpha}(u \cdot \nabla) u=-\nabla \partial^{\alpha} \Pi+\partial^{\alpha}(b \cdot \nabla b)+\nu \Delta \partial^{\alpha} u, \\
\partial_{t} \partial^{\alpha} b+\partial^{\alpha}(u \cdot \nabla) b=\partial^{\alpha}(b \cdot \nabla) u+\eta \Delta \partial^{\alpha} b, \\
\nabla \cdot u=\nabla \cdot b=0,
\end{array}\right.
$$

We multiply by $\partial^{\alpha} u$ and $\partial^{\alpha} b$ both sides of the first two equations in (5.26), respectively, for some integer $|\alpha|>\frac{n}{2}+1$, and integrate by parts over $\mathbb{R}^{n}$ to obtain

$$
\begin{aligned}
& \frac{d}{d t}\left[\left\|\partial^{\alpha} u\right\|_{2}^{2}+\left\|\partial^{\alpha} b\right\|_{2}^{2}\right]+2 \nu\left\|\nabla \partial^{\alpha} u\right\|_{2}^{2}+2 \eta\left\|\nabla \partial^{\alpha} b\right\|_{2}^{2} \\
= & -2\left(\partial^{\alpha}(u \cdot \nabla) u, \partial^{\alpha} u\right)-2\left(\partial^{\alpha}(u \cdot \nabla) b, \partial^{\alpha} b\right) \\
& +2\left(\partial^{\alpha}(b \cdot \nabla) b, \partial^{\alpha} u\right)+2\left(\partial^{\alpha}(b \cdot \nabla) u, \partial^{\alpha} b\right) \\
\triangleq & I_{1}+I_{2}+I_{3}+I_{4} .
\end{aligned}
$$

Since

$$
\left(u \cdot \nabla \partial^{\alpha} u, \partial^{\alpha} u\right)=\left(u \cdot \nabla \partial^{\alpha} b, \partial^{\alpha} b\right)=0,
$$


one verifies that by Morse type estimate

$$
\begin{aligned}
I_{1} & \leq 2\left|\left(\partial^{\alpha}(u \cdot \nabla u), \partial^{\alpha} u\right)\right|=2\left|\left(\partial^{\alpha}(u \cdot \nabla) u-u \cdot \nabla \partial^{\alpha} u, \partial^{\alpha} u\right)\right| \\
& \leq C\left[\|u\|_{H^{|\alpha|}}\|\nabla u\|_{\infty}+\|\nabla u\|_{\infty}\|\nabla u\|_{H^{|\alpha|-1}}\right]\|u\|_{H^{|\alpha|}} \\
& \leq C\|\nabla u\|_{\infty}\|u\|_{H^{|\alpha|}}^{2},
\end{aligned}
$$

and

$$
\begin{aligned}
I_{2} & \leq C\left[\|u\|_{H^{|\alpha|} \mid}\|\nabla b\|_{\infty}+\|\nabla u\|_{\infty}\|\nabla b\|_{H^{s-1}}\right]\|u\|_{H^{|\alpha|}} \\
& \leq C\left[\|\nabla u\|_{\infty}+\|\nabla b\|_{\infty}\right]\left(\|u\|_{H^{|\alpha|}}^{2}+\|b\|_{H^{|\alpha|}}^{2}\right) .
\end{aligned}
$$

On the other hand, in view of

$$
\int_{\mathbb{R}^{n}}\left[(b \cdot \nabla) \partial^{\alpha} b \partial^{\alpha} u+(b \cdot \nabla) \partial^{\alpha} u \partial^{\alpha} b\right] d x=0,
$$

we show that

$$
\begin{aligned}
I_{3}+I_{4}= & \int_{\mathbb{R}^{n}}\left\{\partial^{\alpha}(b \cdot \nabla) b-\left(b \cdot \nabla \partial^{\alpha} b\right)\right\} \partial^{\alpha} u d x \\
& +\int_{\mathbb{R}^{n}}\left\{\partial^{\alpha}(b \cdot \nabla) u-\left(b \cdot \nabla \partial^{\alpha} u\right)\right\} \partial^{\alpha} b d x \\
\leq & C\left[\|\nabla u\|_{\infty}+\|\nabla b\|_{\infty}\right]\left(\|u\|_{H^{|\alpha|}}^{2}+\|b\|_{H^{|\alpha|}}^{2}\right),
\end{aligned}
$$

which implies

$$
\begin{aligned}
& \frac{d}{d t}\left[\left\|\partial^{\alpha} u\right\|_{2}^{2}+\left\|\partial^{\alpha} b\right\|_{2}^{2}\right]+2 \nu\left\|\nabla \partial^{\alpha} u\right\|_{2}^{2}+2 \eta\left\|\nabla \partial^{\alpha} b\right\|_{2}^{2} \\
\leq & C\left[\|\nabla u\|_{\infty}+\|\nabla b\|_{\infty}\right]\left(\|u\|_{H^{|\alpha|}}^{2}+\|b\|_{H^{|\alpha|}}^{2}\right) .
\end{aligned}
$$

In the same way as leading to (5.32), it follows that

$$
\frac{d}{d t}\left[\|u\|_{2}^{2}+\|b\|_{2}^{2}\right]+2 \nu\|\nabla u\|_{2}^{2}+2 \eta\|\nabla b\|_{2}^{2} \leq C\left[\|\nabla u\|_{\infty}+\|\nabla b\|_{\infty}\right]\left(\|u\|_{2}^{2}+\|b\|_{2}^{2}\right) .
$$

(5.32) and (5.33) together with the Gronwall inequality imply

$$
\|(u(t), b(t))\|_{H^{|\alpha|}} \leq\left\|\left(u_{0}, b_{0}\right)\right\|_{H^{|\alpha|}} \exp \left(C \int_{0}^{t}\left[\|\nabla u\|_{\infty}+\|\nabla b\|_{\infty}\right] d s\right) .
$$

Note that

$$
\|(\nabla u, \nabla b)\|_{\infty} \leq C\left(1+\|(\nabla u, \nabla b)\|_{\dot{B}_{\infty, \infty}^{0}}\left(1+\log ^{+}\|(u, b)\|_{H^{|\alpha|}}\right)\right),
$$

and

$$
(\nabla u, \nabla b)=C(\nabla \times u, \nabla \times b)+\mathcal{P}((\nabla \times u, \nabla \times b)),
$$

it follows that

$$
\|(\nabla u, \nabla b)\|_{\infty} \leq C\left(1+\|(\nabla \times u, \nabla \times b)\|_{\dot{B}_{\infty, \infty}^{0}}\left(1+\log ^{+}\|(u, b)\|_{H^{|\alpha|} \mid}\right)\right) .
$$

Let $Z(t)=\log \left(e+\|(u, b)\|_{H^{|\alpha|} \mid}\right)$, then

$$
Z(t) \leq Z(0)+C T+\int_{0}^{t}\|(\nabla \times u, \nabla \times b)\|_{\dot{B}_{\infty, \infty}^{0}} Z(s) d s .
$$


This along with the Gronwall inequality yields

$$
Z(t) \leq(Z(0)+C T) \exp \left(\int_{0}^{t}\|(\nabla \times u, \nabla \times b)\|_{\dot{B}_{\infty, \infty}^{0}} d s\right) .
$$

Hence, we have

$$
\|(u(t), b(t))\|_{H^{|\alpha|}} \leq C\left(\left\|\left(u_{0}, b_{0}\right)\right\|_{H^{|\alpha|}}+e\right)^{A(t)} \exp (C T A(t)), \quad \forall t \in(0, T],
$$

where

$$
A(t)=\int_{0}^{t}\|(\nabla \times u, \nabla \times b)\|_{\dot{B}_{\infty, \infty}^{0}} d s .
$$

Therefore it is possible to extend the strong solution past $T$, see also [36]. The proof of Theorem 5.1 is thus completed.

REMARK 5.3. We can get the same result in the framework of BMO spaces if we substitute (5.34) with

$$
\|(\nabla u, \nabla b)\|_{\infty} \leq C\left(1+\|(\nabla u, \nabla b)\|_{\mathrm{BMO}}\left(1+\log ^{+}\|(u, b)\|_{H^{|\alpha|}}\right)\right) .
$$

Beale-Kato-Majda [1] and Kozono-Taniuchi [18] showed that for $1<r<\infty$,

$$
\|f\|_{\infty} \leq C\left\{1+\|f\|_{\mathrm{BMO}}\left(\log \left(e+\|f\|_{W^{s, r}}\right)\right)\right\}, \quad f \in W^{s, r}, \quad s>\frac{n}{r},
$$

and

$$
\begin{gathered}
\|\nabla u\|_{\infty} \leq C\left\{1+\|\operatorname{rot} u\|_{\mathrm{BMO}}\left(\log \left(e+\|u\|_{W^{s+1, r}}\right)\right)\right\} \\
u \in W^{s+1, r} \quad \text { with } \operatorname{div} u=0 \quad, \quad s>\frac{n}{r}, \\
\|f \nabla g\|_{r} \leq C\left\{\|f\|_{r}\|\nabla g\|_{\mathrm{BMO}}+\|\nabla f\|_{\mathrm{BMO}}\|g\|_{r}\right\}, \\
f, g \in W^{1, r}, \quad \nabla f, \nabla g \in \mathrm{BMO}, \\
\left\|\partial^{\alpha} f \partial^{\beta} g\right\|_{r} \leq C\|f\|_{\mathrm{BMO}}\left\|(-\Delta)^{\frac{|\alpha|+|\beta|}{2}} g\right\|_{r}+C\|g\|_{\mathrm{BMO}}\left\|(-\Delta)^{\frac{|\alpha|+|\beta|}{2}} f\right\|_{r}, \\
f, g \in \mathrm{BMO} \cap W^{|\alpha|+|\beta|, r}, \quad C=C(n, r, \alpha, \beta),
\end{gathered}
$$

By the above inequalities we can derive the regularity criterion in the framework of BMO spaces based on $u$ and $b$. In detail, $(u, b) \in C^{\infty}\left((0, T) \times \mathbb{R}^{n}\right)$ if

$$
\int_{0}^{T}\left[\|u\|_{\mathrm{BMO}}^{2}+\|b\|_{\mathrm{BMO}}^{2}\right] d t<\infty .
$$

In fact, by means of (5.42), (5.43) together with the Hölder inequality, it follows that

$$
\begin{aligned}
\left|\left(\partial^{\alpha}(u \cdot \nabla) u, \partial^{\alpha} u\right)\right| & \leq\left\|\partial^{\alpha}(u \cdot \nabla) u\right\|_{2}\left\|\partial^{\alpha} u\right\|_{2} \\
& \leq\|u\|_{\mathrm{BMO}}\left\|\partial^{|\alpha|+1} u\right\|_{2}\left\|\partial^{\alpha} u\right\|_{2} \\
& \leq C(\nu)\|u\|_{\mathrm{BMO}}^{2}\left\|\partial^{\alpha} u\right\|_{2}^{2}+\frac{\nu}{4}\left\|\partial^{|\alpha|+1} u\right\|_{2}^{2}, \\
\left|\left(\partial^{\alpha}(u \cdot \nabla) b, \partial^{\alpha} b\right)\right| \leq & \|u\|_{\mathrm{BMO}}\left\|\nabla^{\alpha+1} b\right\|_{2}\left\|\partial^{\alpha} b\right\|_{2}+\left\|\nabla^{|\alpha|+1} u\right\|_{2}\|b\|_{\mathrm{BMO}}\left\|\partial^{\alpha} b\right\|_{2} \\
\leq & C(\nu, \eta)\left(\|u\|_{\mathrm{BMO}}^{2}+\|b\|_{\mathrm{BMO}}^{2}\right)\left\|\partial^{|\alpha|} u\right\|_{2}^{2} \\
& +\frac{\nu}{4}\left\|\nabla^{|\alpha|+1} u\right\|_{2}^{2}+\frac{\eta}{4}\left\|\nabla^{|\alpha|+1} b\right\|_{2}^{2},
\end{aligned}
$$




$$
\begin{aligned}
\left|\left(\partial^{\alpha}(b \cdot \nabla) b, \partial^{\alpha} u\right)\right| & \leq\|b\|_{\mathrm{BMO}}\left\|\partial^{|\alpha|+1} b\right\|_{2}\left\|\partial^{\alpha} u\right\|_{2} \\
& \leq C(\eta)\|b\|_{\mathrm{BMO}}^{2}\left\|\partial^{\alpha} u\right\|_{2}^{2}+\frac{\eta}{4}\left\|\partial^{|\alpha|+1} b\right\|_{2}^{2}
\end{aligned}
$$

and

$$
\begin{aligned}
\left|\left(\partial^{\alpha}(b \cdot \nabla) u, \partial^{\alpha} b\right)\right| \leq & \|b\|_{\mathrm{BMO}}\left\|\nabla^{\alpha+1} u\right\|_{2}\left\|\partial^{\alpha} b\right\|_{2}+\left\|\nabla^{|\alpha|+1} b\right\|_{2}\|u\|_{\mathrm{BMO}}\left\|\partial^{\alpha} b\right\|_{2} \\
\leq & C(\nu, \eta)\left(\|u\|_{\mathrm{BMO}}^{2}+\|b\|_{\mathrm{BMO}}^{2}\right)\left\|\partial^{|\alpha|} u\right\|_{2}^{2} \\
& +\frac{\nu}{4}\left\|\nabla^{|\alpha|+1} u\right\|_{2}^{2}+\frac{\eta}{4}\left\|\nabla^{|\alpha|+1} b\right\|_{2}^{2} .
\end{aligned}
$$

Collecting (5.27), (5.45)-(5.48) we easily show the regularity result in the framework of BMO space, see also [37].

\section{Uniqueness and regularity criterion in the framework of mixed space-time}

Besov space. We shall apply Tao's trichotomy method to study the regularity of solutions to the MHD equations.

Localizing the MHD equations (1.1)-(1.3), we obtain the following equations for $j=$ $1,2, \ldots, n$ :

$$
\left\{\begin{array}{l}
\partial_{t} \triangle_{k} u^{j}-\nu \Delta \triangle_{k} u^{j}+\triangle_{k}\left(u^{i} \partial_{i} u^{j}\right)=-\partial_{j} \triangle_{k} \Pi+\triangle_{k}\left(b^{i} \partial_{i} b^{j}\right) \\
\partial_{t} \triangle_{k} b^{j}-\eta \Delta \triangle_{k} b^{j}+\triangle_{k}\left(u^{i} \partial_{i} b^{j}\right)=\triangle_{k}\left(b^{i} \partial_{i} u^{j}\right) \\
\Pi=R_{i} R_{m}\left(u^{i} u^{m}\right)-R_{i} R_{m}\left(b^{i} b^{m}\right) \\
\nabla \cdot u=\nabla \cdot b=0
\end{array}\right.
$$

Multiplying by $\left|\triangle_{k} u^{j}\right|^{r-2} \triangle_{k} u^{j}$ and $\left|\triangle_{k} b^{j}\right|^{r-2} \triangle_{k} b^{j}$ both sides of the first two equations of (6.1), respectively, and integrating with respect to $x$ over $\mathbb{R}^{n}$, one gets

$$
\begin{aligned}
& \frac{1}{r} \frac{d}{d t} \int_{\mathbb{R}^{n}}\left|\triangle_{k} u^{j}\right|^{r} d x+\frac{1}{r} \frac{d}{d t} \int_{\mathbb{R}^{n}}\left|\triangle_{k} b^{j}\right|^{r} d x \\
& +(r-1) \nu \int_{\mathbb{R}^{n}}\left|\nabla \triangle_{k} u^{j}\right|^{2}\left|\triangle_{k} u^{j}\right|^{r-2} d x+(r-1) \eta \int_{\mathbb{R}^{n}}\left|\nabla \triangle_{k} b^{j}\right|^{2}\left|\triangle_{k} b^{j}\right|^{r-2} d x \\
= & -\int_{\mathbb{R}^{n}} \triangle_{k}\left(u^{i} \partial_{i} u^{j}\right)\left|\triangle_{k} u^{j}\right|^{r-2} \triangle_{k} u^{j} d x+\int_{\mathbb{R}^{n}} \triangle_{k}\left(b^{i} \partial_{i} b^{j}\right)\left|\triangle_{k} u^{j}\right|^{r-2} \triangle_{k} u^{j} d x \\
& -\int_{\mathbb{R}^{n}} \partial_{j} \triangle_{k}(\Pi)\left|\triangle_{k} u^{j}\right|^{r-2} \triangle_{k} u^{j} d x-\int_{\mathbb{R}^{n}} \triangle_{k}\left(u^{i} \partial_{i} b^{j}\right)\left|\triangle_{k} b^{j}\right|^{r-2} \triangle_{k} b^{j} d x \\
& +\int_{\mathbb{R}^{n}} \triangle_{k}\left(b^{i} \partial_{i} u^{j}\right)\left|\triangle_{k} b^{j}\right|^{r-2} \triangle_{k} b^{j} d x \\
\triangleq & I_{1}+I_{2}+I_{3}+I_{4}+I_{5} .
\end{aligned}
$$

For the notational convenience, we denote

$$
\sum_{|\lambda-k| \leq m} \triangle_{\lambda} \triangleq \widetilde{\triangle}_{k, m}
$$

From the generalized Bernstein estimates

$$
\int_{\mathbb{R}^{n}}\left|\nabla \triangle_{k} u^{j}\right|^{2}\left|\triangle_{k} u^{j}\right|^{r-2} d x \sim 2^{2 k} \int_{\mathbb{R}^{n}}\left|\triangle_{k} u^{j}\right|^{r} d x
$$


it follows that

$$
\begin{aligned}
& \frac{1}{r} \frac{d}{d t} \int_{\mathbb{R}^{n}}\left[\left|\triangle_{k} u^{j}\right|^{r}+\left|\triangle_{k} b^{j}\right|^{r}\right] d x+2^{2 k} C \int_{\mathbb{R}^{n}}\left[\left|\triangle_{k} u\right|^{r}+\left|\triangle_{k} b\right|^{r}\right] d x \\
\leq & I_{1}+I_{2}+I_{3}+I_{4}+I_{5} .
\end{aligned}
$$

By the Littlewood-Paley trichotomy decomposition, we have by taking $f=u^{i}, g=\partial_{i} u^{j}$

$$
\begin{aligned}
I_{1}= & \int_{\mathbb{R}^{n}} \triangle_{k}\left(u^{i} \partial_{i} u^{j}\right)\left|\triangle_{k} u^{j}\right|^{r-2} \triangle_{k} u^{j} d x \\
= & \sum_{\ell<k-5} \int_{\mathbb{R}^{n}} \triangle_{k}\left(\widetilde{\triangle}_{k, 3} u^{i} \triangle_{\ell}\left(\partial_{i} u^{j}\right)\right)\left|\triangle_{k} u^{j}\right|^{r-2} \triangle_{k} u^{j} d x \\
& +\sum_{\ell<k-5} \int_{\mathbb{R}^{n}} \triangle_{k}\left(\triangle_{\ell} u^{i} \widetilde{\triangle}_{k, 3} \partial_{i} u^{j}\right)\left|\triangle_{k} u^{j}\right|^{r-2} \triangle_{k} u^{j} d x \\
& +\int_{\mathbb{R}^{n}} \triangle_{k}\left(\widetilde{\triangle}_{k, 5} u^{i} \widetilde{\triangle}_{k, 5} \partial_{i} u^{j}\right)\left|\triangle_{k} u^{j}\right|^{r-2} \triangle_{k} u^{j} d x \\
& +\sum_{\ell, \text { or } \ell^{\prime}>k+5} \int_{\mathbb{R}^{n}} \triangle_{k}\left(\triangle_{\ell} u^{i} \triangle_{\ell^{\prime}} \partial_{i} u^{j}\right)\left|\triangle_{k} u^{j}\right|^{r-2} \triangle_{k} u^{j} d x \\
\triangleq & I^{(1)}+I \ell^{(1)}+I I I^{(1)}+I V^{(1)} .
\end{aligned}
$$

We have

$$
\begin{aligned}
I^{(1)} & \lesssim \sum_{\ell<k-5}\left\|\widetilde{\triangle}_{k, 3} u\right\|_{r}\left\|\triangle_{k} u\right\|_{r}^{r-1}\left\|\triangle_{\ell}\left(\partial_{x_{i}} u\right)\right\|_{\infty} \\
& \lesssim\left\|\widetilde{\triangle}_{k, 3} u\right\|_{r}\left\|\triangle_{k} u\right\|_{r}^{r-1} \sum_{\ell<k-5} 2^{\left(\frac{n}{\tilde{r}}+1\right) \ell}\left\|\triangle_{\ell} u\right\|_{\tilde{r}}, \\
I I I^{(1)} & \lesssim\left\|\widetilde{\triangle}_{k, 5} u\right\|_{r}\left\|\triangle_{k} u\right\|_{r}^{r-1} \sum_{\ell=k-5}^{k+5} 2^{\left(\frac{n}{\tilde{r}}+1\right) \ell}\left\|\triangle_{\ell} u\right\|_{\tilde{r}}
\end{aligned}
$$

Since

$$
\triangle_{k}\left(\triangle_{\ell} u^{i} \triangle_{\ell^{\prime}}\left(\partial_{i} u^{j}\right)\right)=\partial_{i}\left(\triangle_{k}\left(\triangle_{\ell} u^{i}\left(\triangle_{\ell^{\prime}} u^{j}\right)\right)\right),
$$

it follows that

$$
\begin{aligned}
I V^{(1)} & \lesssim 2^{k}\left\|\triangle_{k} u\right\|_{\infty}\left\|\triangle_{k} u\right\|_{r}^{r-2} \sum_{\ell, \text { or } \ell^{\prime}>k+5} \sum_{\left|\ell-\ell^{\prime}\right| \leq 3}\left\|\triangle_{\ell} u\right\|_{r}\left\|\triangle_{\ell^{\prime}} u\right\|_{r} \\
& \lesssim 2^{\left(\frac{n}{\tilde{r}}+1\right) k}\left\|\triangle_{k} u\right\|_{\tilde{r}}\left\|\triangle_{k} u\right\|_{r}^{r-2} \sum_{\ell, \text { or } \ell^{\prime}>k+5} \sum_{\left|\ell-\ell^{\prime}\right| \leq 3}\left\|\triangle_{\ell} u\right\|_{r}\left\|\triangle_{\ell^{\prime}} u\right\|_{r} .
\end{aligned}
$$

Now we are in a position to estimate $I I^{(1)}$. Since

$$
\begin{aligned}
& \int_{\mathbb{R}^{n}} \triangle_{\ell} u^{i} \triangle_{k}\left(\widetilde{\triangle}_{k, 3} \partial_{i} u^{j}\right)\left|\triangle_{k} u^{j}\right|^{r-2} \triangle_{k} u^{j} d x=\int_{\mathbb{R}^{n}} \triangle_{\ell} u^{i} \triangle_{k}\left(\partial_{i} u^{j}\right)\left|\triangle_{k} u^{j}\right|^{r-2} \triangle_{k} u^{j} d x \\
= & \int_{\mathbb{R}^{n}} \triangle_{\ell} u^{i} \partial_{i} \triangle_{k} u^{j}\left|\triangle_{k} u^{j}\right|^{r-2} \triangle_{k} u^{j} d x \\
= & \frac{1}{2} \int_{\mathbb{R}^{n}} \triangle_{\ell} u^{i} \partial_{i}\left|\triangle_{k} u^{j}\right|^{2}\left|\triangle_{k} u^{j}\right|^{r-2} d x \\
= & r \int_{\mathbb{R}^{n}}^{\triangle_{\ell} u^{i} \partial_{i}\left(\left|\triangle_{k} u^{j}\right|^{2} \mid\right)^{\frac{r}{2}} d x=0,}
\end{aligned}
$$


and

$$
\begin{aligned}
& \left\|\triangle_{k}\left(\triangle_{\ell} u^{i} \widetilde{\triangle}_{k, 3} \partial_{i} u^{j}\right)-\triangle_{\ell} u^{i} \triangle_{k}\left(\widetilde{\triangle}_{k, 3} \partial_{i} u^{j}\right)\right\|_{p} \lesssim\left\|\triangle_{\ell} \nabla u\right\|_{\infty}\left\|\widetilde{\triangle}_{k, 3} u\right\|_{r} \\
\lesssim & 2^{\left(\frac{n}{\tilde{r}}+1\right) \ell}\left\|\triangle_{\ell} u\right\|_{\tilde{r}}\left\|\widetilde{\triangle}_{k, 3} u\right\|_{r}
\end{aligned}
$$

we easily verify that

$$
\begin{aligned}
I I^{(1)} & \lesssim \sum_{\ell<k-5} \int_{\mathbb{R}^{n}}\left\{\triangle_{k}\left(\triangle_{\ell} u^{i} \widetilde{\triangle}_{k, 3} \partial_{i} u^{j}\right)-\triangle_{\ell} u^{i} \triangle_{k}\left(\widetilde{\triangle}_{k, 3} \partial_{i} u^{j}\right)\right\}\left|\triangle_{k} u^{j}\right|^{r-2} \triangle_{k} u^{j} d x \\
& \lesssim\left\|\widetilde{\triangle}_{k, 3} u\right\|_{r}\left\|\triangle_{k} u\right\|_{r}^{r-1} \sum_{\ell<k-5} 2^{\left(\frac{n}{\tilde{r}}+1\right) \ell}\left\|\triangle_{\ell} u\right\|_{\tilde{r}}
\end{aligned}
$$

Putting estimates of $I^{(1)}, I I^{(1)}, I I I^{(1)}, I V^{(1)}$ into (6.3), one gets

$$
\begin{aligned}
\left|I_{1}\right| \lesssim & \sum_{\ell<k+5} 2^{\left(\frac{n}{\tilde{r}}+1\right) \ell}\left\|\triangle_{\ell} u\right\|_{\tilde{r}}\left\|\triangle_{k} u\right\|_{r}^{r-2} \\
& \times\left[\sum_{m=k-5}^{k+5}\left\|\triangle_{m} u\right\|_{r}^{2}+\sum_{\ell, \text { or } \ell^{\prime}>k+5} \sum_{\left|\ell-\ell^{\prime}\right| \leq 3}\left\|\triangle_{\ell} u\right\|_{r}\left\|\triangle_{\ell^{\prime}} u\right\|_{r}\right] .
\end{aligned}
$$

Secondly, we estimate $I_{4}$

$$
\begin{aligned}
I_{4}= & \int_{\mathbb{R}^{n}} \triangle_{k}\left(u^{i} \partial_{i} b^{j}\right)\left|\triangle_{k} b^{j}\right|^{r-2} \triangle_{k} b^{j} d x \\
= & \sum_{\ell<k-5} \int_{\mathbb{R}^{n}} \triangle_{k}\left(\widetilde{\triangle}_{k, 3} u^{i} \triangle_{\ell}\left(\partial_{i} b^{j}\right)\right)\left|\triangle_{k} b^{j}\right|^{r-2} \triangle_{k} b^{j} d x \\
& +\sum_{\ell<k-5} \int_{\mathbb{R}^{n}} \triangle_{k}\left(\triangle_{\ell} u^{i} \widetilde{\triangle}_{k, 3} \partial_{i} b^{j}\right)\left|\triangle_{k} b^{j}\right|^{r-2} \triangle_{k} b^{j} d x \\
& +\int_{\mathbb{R}^{n}} \triangle_{k}\left(\widetilde{\triangle}_{k, 5} u^{i} \widetilde{\triangle}_{k, 5} \partial_{i} b^{j}\right)\left|\triangle_{k} b^{j}\right|^{r-2} \triangle_{k} b^{j} d x \\
& +\sum_{\ell \text { or } \ell^{\prime}>k+5\left|\ell-\ell^{\prime}\right| \leq 3} \int_{\mathbb{R}^{n}} \triangle_{k}\left(\triangle_{\ell} u^{i} \triangle_{\ell^{\prime}} \partial_{i} b^{j}\right)\left|\triangle_{k} b^{j}\right|^{r-2} \triangle_{k} b^{j} d x \\
\triangleq & I^{(4)}+I I^{(4)}+I I I^{(4)}+I V^{(4)} .
\end{aligned}
$$

It follows that

$$
\begin{aligned}
I^{(4)} & \lesssim \sum_{\ell<k-5}\left\|\widetilde{\triangle}_{k, 3} u\right\|_{r}\left\|\triangle_{k} b\right\|_{r}^{r-1}\left\|\triangle_{\ell}\left(\partial_{x_{i}} b\right)\right\|_{\infty} \\
& \lesssim\left\|\widetilde{\triangle}_{k, 3} u\right\|_{r}\left\|\triangle_{k} b\right\|_{r}^{r-1} \sum_{\ell<k-5} 2^{\left(\frac{n}{\tilde{r}}+1\right) \ell}\left\|\triangle_{\ell} b\right\|_{\tilde{r}} \\
I I I^{(4)} & \lesssim\left\|\widetilde{\triangle}_{k, 5} u\right\|_{r}\left\|\triangle_{k} b\right\|_{r}^{r-1} \sum_{\ell=k-5}^{k+5} 2^{\left(\frac{n}{\tilde{r}}+1\right) \ell}\left\|\triangle_{\ell} b\right\|_{\tilde{r}}
\end{aligned}
$$

Since

$$
\triangle_{k}\left(\triangle_{\ell} u^{i} \triangle_{\ell^{\prime}} \partial_{i} b^{j}\right)=\partial_{i}\left(\triangle_{k}\left(\triangle_{\ell} u^{i} \triangle_{\ell^{\prime}} b^{j}\right),\right.
$$


it follows by the Hölder inequality

$$
\begin{aligned}
I V^{(4)} & \leq C 2^{k}\left\|\triangle_{k} b\right\|_{\infty}\left\|\triangle_{k} b\right\|_{r}^{r-2} \sum_{\ell, \text { or } \ell^{\prime}>k+5} \sum_{\left|\ell-\ell^{\prime}\right| \leq 3}\left\|\triangle_{\ell} u\right\|_{r}\left\|\triangle_{\ell^{\prime}} b\right\|_{r} \\
& \leq C 2^{\left(\frac{n}{\tilde{r}}+1\right) k}\left\|\triangle_{k} b\right\|_{\tilde{r}}\left\|\triangle_{k} b\right\|_{r}^{r-2} \sum_{\ell, \text { or } \ell^{\prime}>k+5} \sum_{\left|\ell-\ell^{\prime}\right| \leq 3}\left\|\triangle_{\ell} u\right\|_{r}\left\|\triangle_{\ell^{\prime}} b\right\|_{r} .
\end{aligned}
$$

Now we are in a position to estimate $I I^{(4)}$. Since

$$
\begin{aligned}
\int_{\mathbb{R}^{n}} \triangle_{\ell} u^{i} \triangle_{k}\left(\widetilde{\triangle}_{k, 3} \partial_{i} b^{j}\right)\left|\triangle_{k} b^{j}\right|^{r-2} \triangle_{k} b^{j} d x & =\int_{\mathbb{R}^{n}} \triangle_{\ell} u^{i} \triangle_{k}\left(\partial_{i} b^{j}\right)\left|\triangle_{k} b^{j}\right|^{r-2} \triangle_{k} b^{j} d x \\
& =r \int_{\mathbb{R}^{n}} \triangle_{\ell} u^{i} \partial_{i}\left(\left|\triangle_{k} b^{j}\right|^{2} \mid\right)^{\frac{r}{2}} d x=0,
\end{aligned}
$$

and

$$
\begin{aligned}
\left\|\triangle_{k}\left(\triangle_{\ell} u^{i} \widetilde{\triangle}_{k, 3} \partial_{i} b^{j}\right)-\triangle_{\ell} u^{i} \triangle_{k}\left(\widetilde{\triangle}_{k, 3} \partial_{i} b^{j}\right)\right\|_{p} & \leq C\left\|\triangle_{\ell} \nabla u\right\|_{\infty}\left\|\widetilde{\triangle}_{k, 3} b\right\|_{r} \\
& \leq C 2^{\left(\frac{n}{\tilde{r}}+1\right) \ell}\left\|\triangle_{\ell} u\right\|_{\tilde{r}}\left\|\widetilde{\triangle}_{k, 3} b\right\|_{r},
\end{aligned}
$$

we easily verify that

$$
\begin{aligned}
I I^{(4)} & \leq \sum_{\ell<k-5} \int_{\mathbb{R}^{n}}\left\{\triangle_{k}\left(\triangle_{\ell} u^{i} \widetilde{\triangle}_{k, 3} \partial_{i} b^{j}\right)-\triangle_{\ell} u^{i} \triangle_{k}\left(\widetilde{\triangle}_{k, 3} b \partial_{i} b^{j}\right)\right\}\left|\triangle_{k} b^{j}\right|^{r-2} \triangle_{k} b^{j} d x \\
& \lesssim\left\|\widetilde{\triangle}_{k, 3} b\right\|_{r}\left\|\triangle_{k} b\right\|_{r}^{r-1} \sum_{\ell<k-5} 2^{\left(\frac{n}{\tilde{r}}+1\right) \ell}\left\|\triangle_{\ell} u\right\|_{\tilde{r}}
\end{aligned}
$$

which implies

$$
\begin{aligned}
\left|I_{4}\right| \leq & C \sum_{\ell<k+5} 2^{\left(\frac{n}{\tilde{r}}+1\right) \ell}\left(\left\|\triangle_{\ell} u\right\|_{\tilde{r}}+\left\|\triangle_{\ell} b\right\|_{\tilde{r}}\right)\left\|\triangle_{k} b\right\|_{r}^{r-2} \\
& \times\left[\sum_{m=k-5}^{k+5}\left[\left\|\triangle_{m} b\right\|_{r}^{2}+\left\|\triangle_{m} u\right\|_{r}^{2}\right]+\sum_{\ell, \text { or } \ell^{\prime}>k+5} \sum_{\left|\ell-\ell^{\prime}\right| \leq 3}\left\|\triangle_{\ell} u\right\|_{r}\left\|\triangle_{\ell^{\prime}} b\right\|_{r}\right] .
\end{aligned}
$$

In the same way as the decomposition of (6.3) and (6.5), we have

$$
I_{j}=I^{(j)}+I I^{(j)}+I I I^{(j)}+I V^{(j)}, \quad j=2,5 .
$$

Moreover, direct computations imply

$$
\begin{aligned}
& \left|I^{(2)}\right| \lesssim\left\|\widetilde{\triangle}_{k, 3} b\right\|_{r}\left\|\triangle_{k} u\right\|_{r}^{r-1} \sum_{\ell<k-5} 2^{\left(\frac{n}{\tilde{r}}+1\right) \ell}\left\|\triangle_{\ell} b\right\|_{\tilde{r}} \\
& \left|I^{(5)}\right| \lesssim\left\|\widetilde{\triangle}_{k, 3} b\right\|_{r}\left\|\triangle_{k} b\right\|_{r}^{r-1} \sum_{\ell<k-5} 2^{\left(\frac{n}{\tilde{r}}+1\right) \ell}\left\|\triangle_{\ell} u\right\|_{\tilde{r}}, \\
& \left|I I I^{(2)}\right| \lesssim\left\|\widetilde{\triangle}_{k, 5} b\right\|_{r}\left\|\triangle_{k} u\right\|_{r}^{r-1} \sum_{\ell=k-5}^{k+5} 2^{\left(\frac{n}{\tilde{r}}+1\right) \ell}\left\|\triangle_{\ell} b\right\|_{\tilde{r}}, \\
& \left|I I I^{(5)}\right| \lesssim\left\|\widetilde{\triangle}_{k, 5} b\right\|_{r}\left\|\triangle_{k} b\right\|_{r}^{r-1} \sum_{\ell=k-5}^{k+5} 2^{\left(\frac{n}{\tilde{r}}+1\right) \ell}\left\|\triangle_{\ell} u\right\|_{\tilde{r}}, \\
& \left|I V^{(2)}\right| \lesssim 2^{\left(\frac{n}{\tilde{r}}+1\right) k}\left\|\triangle_{k} u\right\|_{\tilde{r}}\left\|\triangle_{k} u\right\|_{r}^{r-2} \sum_{\substack{\ell \text { or } \ell^{\prime}>k+5 \\
\left|\ell-\ell^{\prime}\right| \leq 3}}\left\|\triangle_{\ell} b\right\|_{r}\left\|\triangle_{\ell^{\prime}} b\right\|_{r} \\
& \left|I V^{(5)}\right| \lesssim 2^{\left(\frac{n}{\bar{r}}+1\right) k}\left\|\triangle_{k} b\right\|_{\tilde{r}}\left\|\triangle_{k} b\right\|_{r}^{r-2} \sum_{\substack{\ell \text { or } \ell^{\prime}>k+5 \\
\left|\ell-\ell^{\prime}\right| \leq 3}}\left\|\triangle_{\ell} u\right\|_{r}\left\|\triangle_{\ell^{\prime}} b\right\|_{r} .
\end{aligned}
$$


Now we estimate $I I^{(2)}$ and $I I^{(5)}$. It follows by the Hölder inequality that

$$
\begin{aligned}
\left|I I^{(2)}\right| & \leq\left.\sum_{\ell<k-5}\left|\int_{\mathbb{R}^{n}} \triangle_{\ell} b^{i} \triangle_{k} \tilde{\triangle}_{k, 3} \partial_{i} b^{j}\right| \triangle_{k} u^{j}\right|^{r-2} \triangle_{k} u^{j} d x \mid \\
& =\left.\sum_{\ell<k-5} \int_{\mathbb{R}^{n}}\left|\triangle_{\ell} b^{i} \triangle_{k} \partial_{i} b^{j}\right| \triangle_{k} u^{j}\right|^{r-2} \triangle_{k} u^{j} \mid d x \\
& \lesssim \sum_{\ell<k-5}\left\|\triangle_{\ell} b^{i}\right\|_{\infty}\left\|\triangle_{k} \partial_{i} b^{j}\right\|_{r}\left\|\triangle_{k} u^{j}\right\|_{r}^{r-1} \\
& \lesssim\left\|\triangle_{k} b\right\|_{r}\left\|\triangle_{k} u\right\|_{r}^{r-1} \sum_{\ell<k-5} 2^{\frac{n}{\tilde{r}} \ell+k}\left\|\triangle_{\ell} b\right\|_{\tilde{r}}, \\
\left|I I^{(5)}\right| & \leq\left.\sum_{\ell<k-5}\left|\int_{\mathbb{R}^{n}} \triangle_{\ell} b^{i} \triangle_{k} \tilde{\triangle}_{k, 3} \partial_{i} u^{j}\right| \triangle_{k} b^{j}\right|^{r-2} \triangle_{k} b^{j} d x \mid \\
& \lesssim\left\|\triangle_{k} u\right\|_{r}\left\|\triangle_{k} b\right\|_{r}^{r-1} \sum_{\ell<k-5} 2^{\frac{n}{\tilde{r}} \ell+k}\left\|\triangle_{\ell} b\right\|_{\tilde{r}} .
\end{aligned}
$$

Collecting the above estimates yields

$$
\begin{aligned}
\left|I_{2}\right| \lesssim & \sum_{\ell<k+5} 2^{\frac{n}{\tilde{r}} \ell+k}\left\|\triangle_{\ell} b\right\|_{\tilde{r}} \cdot\left\|\triangle_{k} u\right\|_{r}^{r-2}\left[\sum_{m=k-5}^{k+5}\left[\left\|\triangle_{m} u\right\|_{r}^{2}+\left\|\triangle_{m} b\right\|_{r}^{2}\right]\right. \\
& \left.+\sum_{\ell \text { or } \ell^{\prime}>k+5} \sum_{\left|\ell-\ell^{\prime}\right| \leq 3}\left\|\triangle_{\ell} b\right\|_{r}\left\|\triangle_{\ell^{\prime}} b\right\|_{r}\right]
\end{aligned}
$$

and

$$
\begin{aligned}
\left|I_{5}\right| \lesssim & \sum_{\ell<k+5} 2^{\frac{n}{\tilde{r}} \ell+k}\left\|\triangle_{\ell} b\right\|_{\tilde{r}} \cdot\left\|\triangle_{k} b\right\|_{r}^{r-2}\left[\sum_{m=k-5}^{k+5}\left[\left\|\triangle_{m} u\right\|_{r}^{2}+\left\|\triangle_{m} b\right\|_{r}^{2}\right]\right. \\
& \left.+\sum_{\ell \text { or } \ell^{\prime}>k+5} \sum_{\left|\ell-\ell^{\prime}\right| \leq 3}\left\|\triangle_{\ell} b\right\|_{r}\left\|\triangle_{\ell^{\prime}} u\right\|_{r}\right] .
\end{aligned}
$$

In the same way as leading to the above estimates, we easily verify that $I_{3}=\int_{\mathbb{R}^{n}}\left[\triangle_{k} R_{i} R_{m}\left(\partial_{j} u^{i} u^{m}+\partial_{j} u^{m} u^{i}\right)-\triangle_{k} R_{i} R_{m}\left(\partial_{j} b^{i} b^{m}+\partial_{j} b^{m} b^{i}\right)\right]\left|\triangle_{k} u^{j}\right|^{r-2} \triangle_{k} u^{j} d x$ satisfies

$$
\begin{aligned}
\left|I_{3}\right| \leq & C \sum_{\ell<k-5} 2^{\left(\frac{n}{\tilde{r}}+1\right) \ell}\left\|\triangle_{\ell} u\right\|_{\tilde{r}}\left\|\triangle_{k} u\right\|_{r}^{r-2}\left[\sum_{m=k-5}^{k+5}\left\|\triangle_{m} u\right\|_{r}^{2}\right. \\
& \left.+\sum_{\ell \text { or } \ell^{\prime}>k+5} \sum_{\left|\ell-\ell^{\prime}\right| \leq 3}\left\|\triangle_{\ell} u\right\|_{r}\left\|\triangle_{\ell^{\prime}} u\right\|_{r}\right] \\
& +C \sum_{\ell<k-5} 2^{\frac{n}{\tilde{r}} \ell+k}\left\|\triangle_{\ell} b\right\|_{\tilde{r}}\left\|\triangle_{k} u\right\|_{r}^{r-2}\left[\sum_{m=k-5}^{k+5}\left[\left\|\triangle_{m} u\right\|_{r}^{2}+\left\|\triangle_{m} b\right\|_{r}^{2}\right]\right. \\
& \left.+\sum_{\ell \text { or } \ell^{\prime}>k+5} \sum_{\left|\ell-\ell^{\prime}\right| \leq 3}\left\|\triangle_{\ell} b\right\|_{r}\left\|\triangle_{\ell^{\prime}} b\right\|_{r}\right] .
\end{aligned}
$$


By means of the estimates from $I_{1}$ to $I_{5}$, one gets

$$
\begin{aligned}
& \frac{1}{r} \frac{d}{d t}\left\|\triangle_{k} u\right\|_{r}^{r}+(r-1) \nu 2^{2 k}\left\|\triangle_{k} u\right\|_{r}^{r} \\
\leq & C \sum_{\ell<k+5} 2^{\frac{n}{\tilde{r}} \ell+k}\left(\left\|\triangle_{\ell} u\right\|_{\tilde{r}}+\left\|\triangle_{\ell} b\right\|_{\tilde{r}}\right)\left\|\triangle_{k} u\right\|_{r}^{r-2}\left[\sum_{m=k-5}^{k+5}\left[\left\|\triangle_{m} u\right\|_{r}^{2}+\left\|\triangle_{m} b\right\|_{r}^{2}\right]\right. \\
& \left.+\sum_{\ell \text { or } \ell^{\prime}>k+5} \sum_{\left|\ell-\ell^{\prime}\right| \leq 3}\left(\left\|\triangle_{\ell} u\right\|_{r}+\left\|\triangle_{\ell} b\right\|_{r}\right)\left(\left\|\triangle_{\ell^{\prime}} u\right\|_{r}+\left\|\triangle_{\ell^{\prime}} b\right\|_{r}\right)\right] \\
& \frac{1}{r} \frac{d}{d t}\left\|\triangle_{k} b\right\|_{r}^{r}+(r-1) \nu 2^{2 k}\left\|\triangle_{k} b\right\|_{r}^{r} \\
\leq & C \sum_{\ell<k+5} 2^{\frac{n}{\tilde{r}} \ell+k}\left(\left\|\triangle_{\ell} u\right\|_{\tilde{r}}+\left\|\triangle_{\ell} b\right\|_{\tilde{r}}\right)\left\|\triangle_{k} b\right\|_{r}^{r-2}\left[\sum_{m=k-5}^{k+5}\left[\left\|\triangle_{m} u\right\|_{r}^{2}+\left\|\triangle_{m} b\right\|_{r}^{2}\right]\right. \\
& \left.+\sum_{\ell \text { or } \ell^{\prime}>k+5\left|\ell-\ell^{\prime}\right| \leq 3}\left(\left\|\triangle_{\ell} u\right\|_{r}+\left\|\triangle_{\ell} b\right\|_{r}\right)\left(\left\|\triangle_{\ell^{\prime}} u\right\|_{r}+\left\|\triangle_{\ell^{\prime}} b\right\|_{r}\right)\right] .
\end{aligned}
$$

Hence

$$
\begin{aligned}
& \frac{d}{d t}\left(\left\|\triangle_{k} u\right\|_{r}^{2}+\left\|\triangle_{k} b\right\|_{r}^{2}\right)+2^{2 k}\left(\left\|\triangle_{k} u\right\|_{r}^{2}+\left\|\triangle_{k} u\right\|_{r}^{2}\right) \\
\leq & C \sum_{\ell<k-5} 2^{\frac{n}{r} \ell+k}\left(\left\|\triangle_{\ell} u\right\|_{\tilde{r}}+\left\|\triangle_{\ell} b\right\|_{\tilde{r}}\right)\left[\sum_{m=k-5}^{k+5}\left[\left\|\triangle_{m} u\right\|_{r}^{2}+\left\|\triangle_{m} b\right\|_{r}^{2}\right]\right. \\
& +\sum_{\ell \text { or } \ell^{\prime}>k+5} \sum_{\left|\ell-\ell^{\prime}\right| \leq 3}\left(\left\|\triangle_{\ell} u\right\|_{r}+\left\|\triangle_{\ell} b\right\|_{r}\right)\left(\left\|\triangle_{\ell^{\prime}} u\right\|_{r}+\left\|\triangle_{\ell^{\prime}} b\right\|_{r}\right] .
\end{aligned}
$$

Denote

$G_{k}(t)=\sum_{m=k-5}^{k+5}\left[\left\|\triangle_{m} u\right\|_{r}^{2}+\left\|\triangle_{m} b\right\|_{r}^{2}\right]+\sum_{\substack{\ell \text { or } \ell^{\prime}>k+5 \\\left|\ell-\ell^{\prime}\right| \leq 3}}\left(\left\|\triangle_{\ell} u\right\|_{r}+\left\|\triangle_{\ell} b\right\|_{r}\right)\left(\left\|\triangle_{\ell^{\prime}} u\right\|_{r}+\left\|\triangle_{\ell^{\prime}} b\right\|_{r}\right)$.

(6.12) along with the Gronwall inequality implies

$$
\begin{aligned}
\left\|\triangle_{k} u\right\|_{r}^{2}+\left\|\triangle_{k} b\right\|_{r}^{2} \lesssim & e^{-2^{2 k} t}\left(\left\|\triangle_{k} u_{0}(x)\right\|_{r}^{2}+\left\|\triangle_{k} b_{0}(x)\right\|_{r}^{2}\right) \\
& +\sum_{\ell \leq k+5} 2^{\frac{n}{\tilde{r}} \ell+k} e^{-2^{2 k} t} *\left[\left(\left\|\triangle_{\ell} u\right\|_{\tilde{r}}+\left\|\triangle_{\ell} b\right\|_{\tilde{r}}\right) G_{k}(t)\right] .
\end{aligned}
$$

Taking the $L^{\infty}(0, T)$-norm, we have

$$
\begin{aligned}
\left\|\triangle_{k} u\right\|_{L^{\infty} L^{r}}^{2}+\left\|\triangle_{k} b\right\|_{L^{\infty} L^{r}}^{2} \lesssim & \left(\left\|\triangle_{k} u_{0}(x)\right\|_{r}^{2}+\left\|\triangle_{k} b_{0}(x)\right\|_{r}^{2}\right) \\
& +\sum_{\ell \leq k+5} 2^{\frac{n}{\tilde{r}} \ell+k}\left\|G_{k}(t)\right\|_{q^{\prime}}\left(\left\|\triangle_{\ell} u\right\|_{L^{q} L^{\tilde{r}}}+\left\|\triangle_{\ell} b\right\|_{L^{q} L^{\tilde{r}}}\right) \\
\lesssim & \left(\left\|\triangle_{k} u_{0}(x)\right\|_{r}^{2}+\left\|\triangle_{k} b_{0}(x)\right\|_{r}^{2}\right) \\
& +2^{\left(\frac{n}{\tilde{r}}+1-\gamma\right) k}\left\|G_{k}(t)\right\|_{q^{\prime}}\left(\|u\|_{\mathcal{L}^{q} \dot{B}_{\tilde{r}, \infty}^{\gamma}}+\|b\|_{\mathcal{L}^{q} \dot{B}_{\tilde{r}, \infty}^{\gamma}}\right) .
\end{aligned}
$$


On the other hand, we take the norm in $L^{1}(0, T)$ and get

$$
\begin{aligned}
\left\|\triangle_{k} u\right\|_{L^{2} L^{r}}^{2} & +\left\|\triangle_{k} b\right\|_{L^{2} L^{r}}^{2} \\
\leq & \left\|e^{-2^{2 k} t}\right\|_{L_{t}^{1}}\left(\left\|\triangle_{k} u_{0}(x)\right\|_{r}^{2}+\left\|\triangle_{k} b_{0}(x)\right\|_{r}^{2}\right) \\
& +C \sum_{\ell \leq k+5} 2^{\frac{n}{\tilde{r}} \ell+k}\left\|e^{-2^{2 k} t}\right\|_{L_{t}^{1}}\left\|G_{k}(t)\right\|_{q^{\prime}}\left(\left\|\triangle_{\ell} u\right\|_{L^{q} L^{\tilde{r}}}+\left\|\triangle_{\ell} b\right\|_{L^{q} L^{\tilde{r}}}\right) \\
\leq & C 2^{-2 k}\left(\left\|\triangle_{k} u_{0}(x)\right\|_{r}^{2}+\left\|\triangle_{k} b_{0}(x)\right\|_{r}^{2}\right) \\
& +C 2^{\left(\frac{n}{\tilde{r}}-1-\gamma\right) k}\left\|G_{k}(t)\right\|_{q^{\prime}}\left(\|u\|_{\mathcal{L}^{q} \dot{B}_{\tilde{r}, \infty}^{\gamma}}+\|b\|_{\mathcal{L}^{q} \dot{B}_{\tilde{r}, \infty}^{\gamma}}\right) .
\end{aligned}
$$

Multiplying by $2^{2 k s}$ both sides of (6.14) and taking the $\ell^{\frac{\varrho}{2}}$-norm, one gets

$$
\begin{aligned}
\|u\|_{\mathcal{L}_{T}^{\infty} \dot{B}_{r, \varrho}^{s}}^{2}+\|b\|_{\mathcal{L}_{T}^{\infty} \dot{B}_{r, \varrho}^{s}}^{2} \lesssim & \left\|u_{0}(x)\right\|_{\dot{B}_{r, \varrho}^{s}}^{2}+\left\|b_{0}(x)\right\|_{\dot{B}_{r, \varrho}^{s}}^{2}+\left(\|u\|_{\mathcal{L}_{T}^{q} \dot{B}_{\tilde{r}, \infty}^{\gamma}}+\|b\|_{\mathcal{L}_{T}^{q} \dot{B}_{\tilde{r}, \infty}^{\gamma}}\right) \\
& \times\left(\sum_{k} 2^{\left(\frac{n}{\tilde{r}}-\gamma+1+2 s\right) \frac{k \varrho}{2}}\left\|G_{k}(t)\right\|_{L_{t}^{q^{\prime}}}^{\frac{\varrho}{2}}\right)^{\frac{\rho}{2}} .
\end{aligned}
$$

Multiplying by $2^{2 k(s+1)}$ both sides of (6.15) and taking the $\ell^{\frac{\varrho}{2}}$-norm, it follows that

$$
\begin{aligned}
\|u\|_{\mathcal{L}_{T}^{2} \dot{B}_{r, \varrho}^{s+1}}^{2+1}+\|b\|_{\mathcal{L}_{T}^{2} \dot{B}_{r, \varrho}^{s+1}}^{2} \lesssim & \left\|u_{0}(x)\right\|_{\dot{B}_{r, \varrho}^{s}}^{2}+\left\|b_{0}(x)\right\|_{\dot{B}_{r, \varrho}^{s}}^{2}+\left(\|u\|_{\mathcal{L}_{T}^{q} \dot{B}_{\tilde{r}, \infty}^{\gamma}}+\left\|\triangle_{\ell} b\right\|_{\mathcal{L}_{T}^{q} \dot{B}_{\tilde{r}, \infty}^{\gamma}}\right) \\
& \times\left(\sum_{k} 2^{\left(\frac{n}{\tilde{r}}-\gamma+1+2 s\right) \frac{k \varrho}{2}}\left\|G_{k}(t)\right\|_{L_{t}^{q^{\prime}}}^{\frac{\varrho}{2}}\right)^{\frac{\rho}{2}} .
\end{aligned}
$$

Now we are left only with proving the following inequality

$$
\left.\sum_{k} 2^{\left(\frac{n}{\tilde{r}}-\gamma+1+2 s\right) \frac{k \varrho}{2}}\left\|G_{k}(t)\right\|_{L_{t}^{q^{\prime}}}^{\frac{\varrho}{2}}\right)^{\frac{\varrho}{2}} \lesssim\|u\|_{\mathcal{L}_{T}^{\infty} \dot{B}_{r, \varrho}^{s}}^{\frac{2}{q}}\|u\|_{\mathcal{L}_{T}^{2} \dot{B}_{r, \varrho}^{s+1}}^{\frac{2}{q^{\prime}}}+\|b\|_{\mathcal{L}_{T}^{\infty} \dot{B}_{r, \varrho}^{s}}^{\frac{2}{q}}\|b\|_{\mathcal{L}_{T}^{2} \dot{B}_{r, \varrho}^{s+1}}^{\frac{2}{q^{\prime}}} .
$$

Noting

$$
G_{k}(t) \lesssim \sum_{\ell \geq k-5}\left(\left\|\triangle_{\ell} u(t)\right\|_{r}^{2}+\left\|\triangle_{\ell} b\right\|_{r}^{2}\right)
$$

and $\theta \triangleq \frac{n}{\tilde{r}}-\gamma+1+2 s>0$, it follows that

$$
\begin{aligned}
& \left(\sum_{k} 2^{\theta \frac{k \varrho}{2}}\left\|G_{k}(t)\right\|_{L_{t}^{q^{\prime}}}^{\frac{\varrho}{2}}\right)^{\frac{\varrho}{2}} \\
& \lesssim\left(\sum_{k} 2^{\theta \frac{k \varrho}{2}}\left(\sum_{\ell \geq k-5}\left\|\triangle_{\ell} u(t)\right\|_{L_{t}^{\infty} L_{x}^{r}}^{\frac{2}{q}}\left\|\triangle_{\ell} u(t)\right\|_{L_{t}^{2} L_{x}^{r}}^{\frac{2}{q^{\prime}}}\right)^{\frac{\varrho}{2}}\right)^{\frac{2}{\varrho}} \\
& +\left(\sum_{k} 2^{\theta \frac{k \varrho}{2}}\left(\sum_{\ell \geq k-5}\left\|\triangle_{\ell} b(t)\right\|_{L_{t}^{\infty} L_{x}^{r}}^{\frac{2}{q}}\left\|\triangle_{\ell} b(t)\right\|_{L_{t}^{2} L_{x}^{r}}^{\frac{2}{q^{\prime}}}\right)^{\frac{\varrho}{2}}\right)^{\frac{2}{\varrho}} \\
& =\left(\sum_{k} 2^{\theta \frac{k \varrho}{2}}\left(\sum_{\ell \geq-5}\left\|\triangle_{\ell+k} u(t)\right\|_{L_{t}^{\infty} L_{x}^{r}}^{\frac{2}{q}}\left\|\triangle_{\ell+k} u(t)\right\|_{L_{t}^{2} L_{x}^{r}}^{\frac{2}{q^{\prime}}}\right)^{\frac{\varrho}{2}}\right)^{\frac{2}{\varrho}} \\
& +\left(\sum_{k} 2^{\theta \frac{k \varrho}{2}}\left(\sum_{\ell \geq-5}\left\|\triangle_{\ell+k} b(t)\right\|_{L_{t}^{\infty} L_{x}^{r}}^{\frac{2}{q}}\left\|\triangle_{\ell+k} b(t)\right\|_{L_{t}^{2} L_{x}^{r}}^{\frac{2}{q^{\prime}}}\right)^{\frac{\varrho}{2}}\right)^{\frac{2}{\varrho}}
\end{aligned}
$$




$$
\begin{aligned}
& =\left(\sum _ { k } \left(\sum _ { \ell \geq - 5 } \left(2^{-\theta \ell}\left(2^{(k+\ell) s}\left\|\triangle_{\ell+k} u(t)\right\|_{L_{t}^{\infty} L_{x}^{r}}\right)^{\frac{2}{q}}\right.\right.\right. \\
& \left.\left.\times\left(2^{(k+\ell)(s+1)}\left\|\triangle_{\ell+k} u(t)\right\|_{L_{t}^{2} L_{x}^{r}}\right)^{\frac{2}{q^{\prime}}}\right)^{\frac{\rho}{2}}\right)^{\frac{2}{\varrho}} \\
& +\left(\sum _ { k } \left(\sum _ { \ell \geq - 5 } \left(2^{-\theta \ell}\left(2^{(k+\ell) s}\left\|\triangle_{\ell+k} b(t)\right\|_{L_{t}^{\infty} L_{x}^{r}}\right)^{\frac{2}{q}}\right.\right.\right. \\
& \left.\left.\times\left(2^{(k+\ell)(s+1)}\left\|\triangle_{\ell+k} b(t)\right\|_{L_{t}^{2} L_{x}^{r}}\right)^{\frac{2}{q^{\prime}}}\right)^{\frac{\varrho}{2}}\right)^{\frac{2}{\varrho}},
\end{aligned}
$$

where we used the fact

$$
(k+\ell) \theta=(k+\ell)\left(2-\frac{2}{q}+2 s\right)=(k+\ell)\left(2 s+\frac{2}{q^{\prime}}\right) .
$$

When $\varrho \geq 2$, by the Minkowski inequality we have

$$
\begin{aligned}
& \left(\sum_{k} 2^{\theta \frac{k \varrho}{2}}\left\|G_{k}(t)\right\|_{L_{t}^{q^{\prime}}}^{\frac{\varrho}{2}}\right)^{\frac{\varrho}{2}} \\
\lesssim & \left.\left(\sum_{\ell \geq-5} 2^{-\theta \ell}\left(\sum_{k} 2^{k s \varrho}\left\|\triangle_{k} u(t)\right\|_{L_{t}^{\infty} L_{x}^{r}}^{\varrho}\right)^{\frac{2}{q}}\left(\sum_{k} 2^{k(s+1) \varrho}\left\|\triangle_{k} u(t)\right\|_{L_{t}^{2} L_{x}^{r}}^{\varrho}\right)^{\frac{1}{q^{\prime}}}\right)\right)^{\frac{2}{\varrho}} \\
& \left.+\left(\sum_{\ell \geq-5} 2^{-\theta \ell}\left(\sum_{k} 2^{k s \varrho}\left\|\triangle_{k} b(t)\right\|_{L_{t}^{\infty} L_{x}^{r}}^{\varrho}\right)^{\frac{2}{q}}\left(\sum_{k} 2^{k(s+1) \varrho}\left\|\triangle_{k} b(t)\right\|_{L_{t}^{2} L_{x}^{r}}^{\varrho}\right)^{\frac{1}{q^{\prime}}}\right)\right)^{\frac{2}{\varrho}} \\
\lesssim & \|u(t)\|_{\mathcal{L}_{t}^{\infty} \dot{B}_{r, \varrho}^{s}}^{\frac{2}{q}}\|u(t)\|_{\mathcal{L}_{t}^{2} \dot{B}_{r, \varrho}^{s+\rho}}^{\frac{2}{q^{\prime}}}+\|b(t)\|_{\mathcal{L}_{t}^{\infty} \dot{B}_{r, \varrho}^{s}}^{\frac{2}{q}}\|b(t)\|_{\mathcal{L}_{t}^{2} \dot{B}_{r, \varrho}^{s+1}}^{\frac{2}{q^{\prime}}}
\end{aligned}
$$

On the other hand, for $\varrho \leq 2$, it follows that by $L^{\frac{\varrho}{2}} \hookrightarrow L^{1}$

$$
\begin{aligned}
& \left(\sum_{k} 2^{\theta \frac{k \varrho}{2}}\left\|G_{k}(t)\right\|_{L_{t}^{q^{\prime}}}^{\frac{\rho}{2}}\right)^{\frac{\varrho}{2}} \\
\lesssim & \left(\sum_{k} 2^{\theta \frac{k \varrho}{2}} \sum_{\ell \geq k-5}\left\|\triangle_{\ell} u(t)\right\|_{L_{t}^{\infty} L_{x}^{r}}^{\frac{\varrho}{q}}\left\|\triangle_{\ell} u(t)\right\|_{L_{t}^{2} L_{x}^{r}}^{\frac{\varrho}{q^{\prime}}}\right)^{\frac{2}{\varrho}} \\
& +\left(\sum_{k} 2^{\theta \frac{k \varrho}{2}} \sum_{\ell \geq k-5}\left\|\triangle_{\ell} b(t)\right\|_{L_{t}^{\infty} L_{x}^{r}}^{\frac{\varrho}{q}}\left\|\triangle_{\ell} b(t)\right\|_{L_{t}^{2} L_{x}^{r}}^{\frac{\varrho}{q^{\prime}}}\right)^{\frac{2}{\varrho}} \\
\lesssim & \left(\sum_{\ell>-5} 2^{-\theta \frac{\ell \varrho}{2}}\left(\sum_{k}\left\|\triangle_{k} u(t)\right\|_{L_{t}^{\infty} L_{x}^{r}}^{\varrho}\right)^{\frac{1}{q}}\left(\sum_{k} 2^{k(s+1) \varrho}\left\|\triangle_{k} u(t)\right\|_{L_{t}^{2} L_{x}^{r}}^{\varrho}\right)^{\frac{1}{q^{\prime}}}\right)^{\frac{2}{\varrho}} \\
+ & \left(\sum_{\ell>-5} 2^{-\theta \frac{\ell \varrho}{2}}\left(\sum_{k}\left\|\triangle_{k} b(t)\right\|_{L_{t}^{\infty} L_{x}^{r}}^{\varrho}\left(\sum_{k}^{\frac{1}{q}} 2^{k(s+1) \varrho}\left\|\triangle_{k} b(t)\right\|_{L_{t}^{2} L_{x}^{r}}^{\varrho}\right)^{\frac{1}{q^{\prime}}}\right)^{\frac{2}{\varrho}}\right. \\
\lesssim & \|u(t)\|_{\mathcal{L}_{t}^{\infty} \dot{B}_{r, \varrho}^{s}}^{\frac{2}{q}}\|u(t)\|_{\mathcal{L}_{t}^{2} \dot{B}_{r, \varrho}^{s+1}}^{\frac{2}{q^{\prime}}}+\|b(t)\|_{\mathcal{L}_{t}^{\infty} \dot{B}_{r, \varrho}^{s}}^{\frac{2}{q}}\|b(t)\|_{\mathcal{L}_{t}^{2} \dot{B}_{r, \varrho}^{s+1}}^{\frac{2}{q^{\prime}}} \cdot
\end{aligned}
$$


Since

$$
\begin{gathered}
\|u(t)\|_{\mathcal{L}_{t}^{q} \dot{B}_{\tilde{r}, \infty}^{\gamma}}\|u(t)\|_{\mathcal{L}_{t}^{\infty} \dot{B}_{r, \varrho}^{s}}^{\frac{2}{q}}\|u(t)\|_{\mathcal{L}_{t}^{2} \dot{B}_{r, \varrho}^{s+1}}^{\frac{2}{q^{\prime}}} \\
\lesssim\|u(t)\|_{\mathcal{L}_{t}^{q} \dot{B}_{\tilde{r}, \infty}^{\gamma}}^{q}\|u(t)\|_{\mathcal{L}_{t}^{\infty} \dot{B}_{r, \varrho}^{s}}^{2}+\frac{1}{2}\|u(t)\|_{\mathcal{L}_{t}^{2} \dot{B}_{r, \varrho}^{s+1}}^{2},
\end{gathered}
$$

and

$$
\begin{gathered}
\|b(t)\|_{\mathcal{L}_{t}^{q} \dot{B}_{\tilde{r}, \infty}^{\gamma}}^{\gamma}\|b(t)\|_{\mathcal{L}_{t}^{\infty} \dot{B}_{r, \varrho}^{s}}^{\frac{2}{q}}\|b(t)\|_{\mathcal{L}_{t}^{2} \dot{B}_{r, \varrho}^{s+1}}^{\frac{2}{q^{\prime}}} \\
\lesssim\|b(t)\|_{\mathcal{L}_{t}^{q} \dot{B}_{\tilde{r}, \infty}^{\gamma}}^{q}\|b(t)\|_{\mathcal{L}_{t}^{\infty} \dot{B}_{r, \varrho}^{s}}^{2}+\frac{1}{2}\|b(t)\|_{\mathcal{L}_{t}^{2} \dot{B}_{r, \varrho}^{s+1}}^{2},
\end{gathered}
$$

we can derive the nonlinear estimate

$$
\begin{aligned}
& \|u(t)\|_{\mathcal{L}_{t}^{\infty} \dot{B}_{r, \varrho}^{s}}^{2}+\|u(t)\|_{\mathcal{L}_{t}^{2} \dot{B}_{r, \varrho}^{s+1}}^{2}+\|b(t)\|_{\mathcal{L}_{t}^{\infty} \dot{B}_{r, \varrho}^{s}}^{2}+\|b(t)\|_{\mathcal{L}_{t}^{2} \dot{B}_{r, \varrho}^{s+1}}^{2} \\
\lesssim & \left\|u_{0}(x)\right\|_{\dot{B}_{r, \varrho}^{s}}^{2}+\left\|b_{0}(x)\right\|_{\dot{B}_{r, \varrho}^{s}}^{2}+\left(\|u(t)\|_{\mathcal{L}_{t}^{q} \dot{B}_{\tilde{r}, \infty}^{\gamma}}^{q}+\|b(t)\|_{\mathcal{L}_{t}^{q} \dot{B}_{\tilde{r}, \infty}^{\gamma}}^{q}\right) \\
& \times\left(\|u(t)\|_{\mathcal{L}_{t}^{2} \dot{B}_{r, \varrho}^{s+1}}^{2}+\|b(t)\|_{\mathcal{L}_{t}^{2} \dot{B}_{r, \varrho}^{s+1}}^{2}\right) .
\end{aligned}
$$

From the above discussion we finally obtain the following result:

ThEOREM 6.1. Let $\left(u_{0}(x), b_{0}(x)\right) \in L^{2}\left(\mathbb{R}^{n}\right)$ with $\nabla \cdot u_{0}=\nabla \cdot b_{0}=0$, and suppose that $(u, b) \in L^{\infty}\left((0, T) ; L^{2}\left(\mathbb{R}^{n}\right)\right) \cap L^{2}\left((0, T) ; \dot{H}^{1}\left(\mathbb{R}^{n}\right)\right)$ is a Leray-Hopf weak solution which satisfies the energy inequality (6.1). Assume that there exists a $(q, \tilde{r}) \in \Lambda_{\gamma}$ with $q \neq \infty$ such that $(u, b) \in \mathcal{L}_{t}^{q} \dot{B}_{\tilde{r}, \infty}^{\gamma}$, Then the solution $(u, b)$ is smooth on $(0, T]$.

\section{References}

[1] J. T. Beale, T. Kato and A. Majda, Remarks on breakdown of smooth solutions for the $3 D$ Euler equations, Comm. Math. Phys. 94 (1984), 61-66.

[2] H. Beirão Da Veiga, Concerning the regularity problem for the solutions of Navier-Stokes equations, C. R. Acad. Sci. Paris 321 (1995), 405-408.

[3] J. Bergh and J. Löfström, Interpolation Spaces, Springer-Verlag, 1976.

[4] L. C. Berselli and G. P. Galdi, Regularity criteria involving the pressure for the weak solutions to the Navier-Stokes equations, Proc. Amer. Math. Soc. 130 (2002), 3585-3595.

[5] R. E. Caflisch, I. Klapper and G. Steele, Remarks on singularities, dimension and energy dissipation for the ideal hydrodynamics and MHD, Comm. Math. Phys. 184 (1997), 443455.

[6] M. Cannone, Ondelettes, Paraproduits et Navier-Stokes, Diderot Ed., Paris, 1995.

[7] M. Cannone, Harmonic analysis tools for solving the incompressible Navier-Stokes equations, in: Handbook of Mathematical Fluid Dynamics, Vol. 3, S. Friedlander and D. Serre (eds.), Elsevier, 2004, 161-244.

[8] E. Casella, P. Secchi and P. Trebeschi, Global classical solutions for MHD system, J. Math. Fluid Mech. 5 (2003), 70-91.

[9] J. Y. Chemin, Perfect Incompressible Fluids, Oxford Lecture Series in Mathematics and its Applications 14, Oxford University Press, New York, 1998. 
[10] J. Y. Chemin, Théorèmes d'unicité pour le système de Navier-Stokes tridimensionnel, J. d'Analyse Math. 77 (1999), 27-50.

[11] J. Y. Chemin and N. Lerner, Flot de champs de vecteurs non lipschitziens et équations de Navier-Stokes, J. Differential Equations 121 (1995), 314-328.

[12] G. Duvaut and J. L. Lions, Inéquations en thermoelasticité et magnétohydrodynamique, Arch. Rational Mech. Anal. 46 (1972), 241-279.

[13] E. B. Fabes, B. F. Jones and N. M. Rivière, The initial value problem for the Navier-Stokes equations with data in $L^{p}$, Arch. Rational Mech. Anal. 45 (1972), 222-240.

[14] H. Fujita and T. Kato, On the Navier-Stokes initial value problem 1, Arch. Rational Mech. Anal. 16 (1964), 269-315.

[15] Y. Giga, Solutions for semilinear parabolic equations in $L^{p}$ and regularity of weak solutions of the Navier-Stokes system, J. Differential Equations 61 (1986), 186-212.

[16] T. Kato, Strong $L^{p}$-solutions of the Navier-Stokes equation in $\mathbb{R}^{m}$, with applications to weak solutions, Math. Z. 187 (1984), 471-480.

[17] H. Kozono, On well-posedness of the Navier-Stokes equations, in: Mathematical Fluid Mechanics, Recent Results and Open Questions, J. Neustupa and P. Penel (eds.), Birkhäuser Verlag, 2001, 207-278.

[18] H. Kozono and Y. Taniuchi, Bilinear estimates in BMO and Navier-Stokes equations, Math. Z. 235 (2000), 191-200.

[19] H. Kozono, T. Ogawa and Y. Taniuchi, The critical Sobolev inequalities in Besov spaces and regularity criterion to some semi-linear evolution equations, Math. Z. 242 (2002), 251-278.

[20] O. A. Ladyzhenskaya, On the Mathematical Theory of the Incompressible Fluid, 2nd ed., Gordon and Breach, New York, 1969.

[21] P. G. Lemarié-Rieusset, Recent Developments in the Navier-Stokes Problem, Chapman \& Hall/CRC, 2002.

[22] J. Leray, Sur le mouvement d'un liquide visqueux emplissant l'espace, Acta Math. 64 (1934), 193-284.

[23] Y. Meyer, Wavelets and Operators, Cambridge University Press, 1992.

[24] K. Masuda, Weak solutions of Navier-Stokes equations, Tohoku Math. J. 36 (1984), 623646 .

[25] C. Miao, Time-space estimates of solutions to general semilinear parabolic equations, Tokyo J. Math. 24 (2001), 245-276.

[26] C. Miao, Harmonic Analysis and Applications to Partial Differential Equations, 2nd ed., Science Press, Beijing, 2004.

[27] C. Miao and B. Zhang, The Cauchy problem for the semilinear parabolic equations in Besov spaces, Houston J. Math. 30 (2004), 829-878.

[28] F. Planchon, Sur une inégalité de type Poincaré, C. R. Acad. Sci. Paris 330 (2000), 21-23.

[29] F. Planchon, An extension of the Beale-Kato-Majda criterion for the Euler equations, Comm. Math. Phys. 232 (2003), 319-326.

[30] M. Sermange and R. Temam, Some mathematical questions related to the MHD equations, Comm. Pure Appl. Math. 36 (1983), 635-664.

[31] J. Serrin, The initial value problem for Navier-Stokes equations, in: Non-Linear Problems, Univ. Wisconsin Press, R. E. Langer (ed.), 1963, 69-98.

[32] E. M. Stein, Singular Integrals and Differentiability Properties of Functions, Princeton University Press, 1970. 
[33] J. Wu, Regularity results for weak solutions of the 3D MHD equations, Discrete Cont. Dynam. Systems 10 (2004), 543-556.

[34] H. Triebel, Theory of Function Spaces, Springer-Verlag, 1983.

[35] W. von Wahl, The Equations of Navier-Stokes and Abstract Parabolic Equations, Vieweg Verlag, Braunschweig, Wiesbaden, 1985.

[36] B. Yuan, Blow up criterion of smooth solution to the MHD equations in Besov Spaces, J. Systems Sci. Complexity 18 (2005), 277-284.

[37] B. Yuan, On the blow-up criterion of smooth solutions to the MHD system in BMO space, Acta Math. Appl. Sinica English Series 22 (2006), 413-418.

[38] Z. Zhang and X. Liu, On the blow-up criterion of smooth solutions to the 3D ideal MHD equations, Acta Math. Appl. Sinica 20 (2004), 695-700.

[39] Z. Zhang and Q. Chen, Space-time estimates in Besov spaces and the Navier-Stokes equations, preprint, 2005, 19 pp. 
